\title{
Operator agency in process intervention: tampering versus application of tacit knowledge
}

\author{
P. Van Gestel ${ }^{1}$ D. J. Pons ${ }^{1}$ V. Pulakanam ${ }^{2}$
}

Received: 24 June 2014/ Accepted: 5 March 2015/Published online: 1 April 2015

(C) The Author(s) 2015. This article is published with open access at Springerlink.com

\begin{abstract}
Statistical process control (SPC) theory takes a negative view of adjustment of process settings, which is termed tampering. In contrast, quality and lean programmes actively encourage operators to acts of intervention and personal agency in the improvement of production outcomes. This creates a conflict that requires operator judgement: How does one differentiate between unnecessary tampering and needful intervention? Also, difficult is that operators apply tacit knowledge to such judgements. There is a need to determine where in a given production process the operators are applying tacit knowledge, and whether this is hindering or aiding quality outcomes. The work involved the conjoint application of systems engineering, statistics, and knowledge management principles, in the context of a case study. Systems engineering was used to create a functional model of a real plant. Actual plant data were analysed with the statistical methods of ANOVA, feature selection, and link analysis. This identified the variables to which the output quality was most
\end{abstract}

D. J. Pons

dirk.pons@canterbury.ac.nz

P. Van Gestel

patrick.vangestel@pg.canterbury.ac.nz

V. Pulakanam

venkat.pulakanam@canterbury.ac.nz

1 Department of Mechanical Engineering, University of Canterbury, Private Bag 4800, Christchurch 8020, New Zealand

2 School of Business and Economics, University of Canterbury, Christchurch, New Zealand sensitive. These key variables were mapped back to the functional model. Fieldwork was then directed to those areas to prospect for operator judgement activities. A natural conversational approach was used to determine where and how operators were applying judgement. This contrasts to the interrogative approach of conventional knowledge management. Data are presented for a case study of a meat rendering plant. The results identify specific areas where operators' tacit knowledge and mental model contribute to quality outcomes and untangles the motivations behind their agency. Also evident is how novice and expert operators apply their knowledge differently. Novices were focussed on meeting throughput objectives, and their incomplete understanding of the plant characteristics led them to inadvertently sacrifice quality in the pursuit of productivity in certain situations. Operators' responses to the plant are affected by their individual mental models of the plant, which differ between operators and have variable validity. Their behaviour is also affected by differing interpretations of how their personal agency should be applied to the achievement of production objectives. The methodology developed here is an integration of systems engineering, statistical analysis, and knowledge management. It shows how to determine where in a given production process the operator intervention is occurring, how it affects quality outcomes, and what tacit knowledge operators are using. It thereby assists the continuous quality improvement processes in a different way to SPC. A second contribution is the provision of a novel methodology for knowledge management, one that circumvents the usual codification barriers to knowledge management.

Keywords Production engineering - Continuous improvement · Lean · Knowledge management - Funnel experiment $\cdot$ Psychology 


\section{Introduction}

The central premise of statistical process control (SPC) is that operators should refrain from adjusting the process providing the part-to-part variability produced by a stable process is within the control limits. To needlessly adjust such a process is to tamper with it (Deming 1986). Yet that assumes that the process genuinely is stable, which is not always the case. In general, a process may have episodes of stability punctuated by instability, and it is not always possible, except in hindsight, for operators to determine when the transitions are occurring. Furthermore, it is impractical to control every variable within a production process, and everything that is not being controlled may be changed by operators. Indeed, a quality culture expects operators to be taking initiative to improve quality and productivity in their work area, hence quality circles and kaizens. Consequently, there are conflicting organisational forces that discourage operator intervention in some situations and encourage it in others. This leads to incongruence, and difficulty in knowing how to handle the borderline processes where stability is weak, or the variable is not designated for active control.

Thus, there are key questions in managing complex manufacturing operations. How does one differentiate between situations where tampering is inadvisable versus those where adjustment is necessary? What situations require manual intervention, relying on operator skill and experience? If operator skill and experience is called for, then what exactly is the skill required, and how do operators acquire it? This last question pertains to how tacit knowledge of operators is developed, and how they use in decision-making. This paper explores these issues by developing a method for identifying where operators are intervening in the plant, and what tacit knowledge they are using. Doing so provides a means to differentiate helpful and unhelpful operator intervention.

\section{Literature}

\section{Tampering}

The purpose of industry is to make profit, and hence, it needs to ensure that the processes it uses are sufficient for the intended outcomes (hence quality) and economically viable. To this end, the control chart was developed by Shewhart for attaining economic control of quality of manufactured product through the establishment of control limits. These indicate at every stage in the production process, from raw material to finished product, when the quality of product is varying more than is economically desirable (Shewhart 1931). Situations that decrease the production economics include production of defects, overworking the product to a quality beyond that which is fit for purpose. There is also the production of waste to consider, hence lean (Pearce and Pons 2013). At the same time, there is variability in all production processes, and control limits represent to the operator how much variation is acceptable before intervention is required. In Shewhart thinking, a process should not be adjusted while it is within the control limits.

The action of adjusting a process while it is still within control limits is considered tampering. It is expected that losses will be incurred if a stable system is tampered with. Deming used an elegant analogy, in the form of the funnel experiment, to demonstrate the magnitude of these losses (Deming 1986). This simple experiment has profoundly shaped attitudes to quality ever since. According to this construct, operators must desist from adjusting a process. They should permit a degree of variability in quality measures and only intervene when the variables exceed the statistically determined control limits. Many variations on the funnel theme have emerged to introduce the statistical concepts to students, workers, and supervisors in business and industry (Alloway 1994; Coleman 1999; Hanna 2010; Krehbiel 1994; Olsen 2007; Schall 2012; Sparks and Field 2000).

Although the funnel experiment has proved to be a versatile and adaptable tool to introduce the SPC control charting technique, in practice, the required condition of process stability is often not met. Trended and regularly adjusted processes are semi-stable and are common in manufacturing industries. A process can be semi-stable because of factors such as tool wear, material replenishment, or regular maintenance. In such situations, interpreting the Shewhart control chart becomes more difficult, such that frequent adjustments might be necessary (Xie et al. 2001, 2002). It has been shown that periodic adjustment of a trending process is beneficial (Xie et al. 2002). Another way, though perhaps semantically contrived, is to differentiate between correction and corrective action, and treat them differently (Davis 2000). From this perspective, avoiding tampering does not mean avoiding fixing problems. A worn tool is a problem that needs fixing, a correction needs to be made, but it does not mean that corrective action is required on the process itself. However, it is not clear how an operator would reliably differentiate between 'correction' and 'corrective action' on the spot. This would require an element of judgement on the part of the operator.

\section{Intervention}

Also, although the idealised funnel experiment is highly relevant to the parts manufacturing industries, it is 
unrealistic for the process industries where the true process mean is usually drifting as a result of some uncontrollable disturbances. In this situation, a policy that actively controls the process to the target will often result in a substantial reduction in the output variance (MacGregor 1990). This implies that an automated process governed by a feedback loop actively controlling the process has to decide when to react and when not to react; do not react when the process is on target, but react when deviance is detected. Methods have been developed to monitor a process in the presence of feedback rules (Singer and Ben Gal 2007), as well as using feedback rules to help detect, explain, and prevent tampering with the processes (Georgantzas and Katsamakas 2008).

The idea of tampering is based on the premise that a production process has a consistent natural random variability, i.e. that successive runs all follow the same underlying statistics. This is a simplistic premise and only applicable to simple production situations. Realistic production plants have many more variables than can possibly be given a SPC treatment. It is only practical to monitor key variables, and the rest are left to inspection, operator common sense, or are covert. Variability intrudes because of the complex interaction between the operators, batches of input materials, and changing condition of the plant. Regarding the latter, production processes can flip between two or more states. For example, backlash in a mechanical control system will give two different states, likewise any process involving hysteresis. Or a night shift may be operating a plant with different load characteristics to the day shift. Defining the control limits by averaging the very different system states will result in sub-optimal quality outputs. Instead, operators in such situations may be better advised to deliberately intervene when the system changes state. So, while Deming's funnel experiment is well established, as are Shewhart control charts, the underlying principles are idealised since not all processes have the requisite level of long-term stability.

\section{Operator agency}

There is also a sociotechnical interaction to consider in the way that operators are motivated to excellence, and how that motivation arises in the plant setting. It is impractical to control every variable in a plant: the level of output control required would simply be unachievable in most practical settings of reasonable complexity. Also, that level of control imposes a psychological external locus of control and consequently is damaging to worker motivation and hence contrary to quality systems. Operators are not simply passive automatons or units of labour, at least not in high-value manufacturing situations. They bring their own motivation to work and are encouraged by other quality processes, namely continuous quality improvement, to be active agents for change and improvement. Consequently, the concept of personal agency becomes an important consideration. This is a psychology concept, and there appears to be no representation yet in the production control literature. High levels of output control arising from SPC are contradictory to the expectations that operators will exhibit innovation and incongruence arises. This may put the operator in a position of cognitive distress, such that a judgement has to be made on which way to proceed: to avoid interfering with the process or to intervene. In reality, operators make these decisions throughout their working day. Even if SPC is rigidly adhered to one part of the plant, there are still a myriad of other variables that are under operator control and which need adjustment.

\section{Tacit knowledge}

Judgement actions (whether or not to intervene) and the actual interventions are based on the operators' skill, which is a function of knowledge. That knowledge is invariably obtained, not from a formal study process, but by experience in the form of over long periods of observation of how the plant behaves. Consequently, operators acquire knowledge about the plant, and each builds for him or herself a mental model of how the plant behaves. This is not necessarily entirely accurate, but, nonetheless, it is the basis for their decision-making and problem-solving acts of agency. Much of the mental model is tacit knowledge that is difficult to consciously articulate. All the same, this knowledge is valuable as a source of process improvements, especially as control charts only apply to a small number of key variables within a production process. Firms find it important to use the tacit knowledge of operators as a part of the improvement cycle, to create new knowledge, obtain competitive advantage, and stay in business (Nonaka 1991; Rowley 1999). That new knowledge could be directed at better production methods just as much as new products. Hence, the application of knowledge management $(\mathrm{KM})$ is at least as important as the prevention of tampering. The knowledge management process consists of a cycle of acquisition of knowledge, dissemination, and the eventual application to a new setting (Jasimuddin 2012). Knowledge has been classified as being either explicit or tacit (Polanyi 1958). Where explicit knowledge is readily codified and transmittable, tacit knowledge has a personal quality which makes it hard to formalise and communicate (Nonaka 1994). Although the validity of the concept of tacit knowledge has been contested (Perraton and Tarrant 2007; Schmidt 2012), it is, nonetheless, a useful concept for the knowledge residing in personnel that is difficult to capture in standard operating procedures (SOP). 
The elusiveness of tacit knowledge derives from at least three reasons: (1) we ourselves are not fully aware of our own tacit knowledge, since it resides in the subconscious; (2) we do not have to make our own tacit knowledge explicit in order to use it ourselves; and (3) we may be reluctant to divulge it because of the risk of losing power and competitive advantage by making it explicit (Stenmark 2000). Consequently, it is difficult to extract tacit knowledge from people (Desouza 2003), and therefore, the process of making it explicit can be effortful regarding time and money (Hamieza and Amirreza 2012). Computer solutions have been sought to reduce the effort necessary to store knowledge and to help personnel to access relevant information (McNaught and Chan 2011). However, the use of technology alone for sharing knowledge is inadequate for competitive advantage, since it also requires social interaction and shared experiences (D'Eredita and Barreto 2006; Goffin and Koners 2011; Johannessen et al. 2001; Stenmark 2000).

So, the knowledge management perspective suggests that operators have tacit knowledge that is important in determining the quality of the production outputs. They are expected to be active agents for quality, by using their knowledge to adjust the production processes for better quality outputs. This tacit knowledge is unable to be fully represented on control charts and standard operating procedures.

\section{Contrasting perspectives}

While the generally accepted wisdom is that operators should not tamper with stable processes, the production reality is more complex. The concept of tampering may be valid for processes that have consistent statistical behaviour, and Shewhart control charts are an excellent way for helping operators understand that they should not tamper with such processes. However, there are many processes that do not have this underlying physical stability, and in which case, it becomes necessary for operators to intervene in the process. Even a stable process is that way only for certain periods and will eventually require operator intervention. Furthermore, in an organisational culture of continuous quality improvement, operators are actively encouraged to apply their tacit knowledge to change the processes for the better, i.e. to apply personal agency. So, operators are in a conflicted situation.

The difficulty is knowing when intervention is warranted. What really is the difference between tampering and application of tacit knowledge? That this is a real issue is apparent from considering the way that quality and lean manufacturing systems emphasise empowerment of operators. One cannot empower the worker to apply his (her) tacit judgement to improve the production process, while simultaneously preventing this by an injunction not to tamper. The issue is the difficulty of identifying whether an intervention is misguided tampering or insightful application of tacit knowledge. In both cases, we are dealing with a class of operator actions that do not fit into standard operating procedures, i.e. they are not readily formalised into explicit work instructions.

Thus, there are two competing regimes: the preservation of the status quo by use of standard operating procedures and control charts with their no-deviation and no-tampering rules vs. the competitive renewal of the organisation by empowerment of workers to be active agents for innovative process improvements. How is an organisation to balance these competing strategic objectives? Executives may be in the easy position of saying they want both, but operational managers have the difficult task of motivating subordinates for both activities, and operators have a complex judgement to make about which approach to take in a situation that is suddenly presented to them. Bear in mind that operators usually have to make the decision on the spot, as it is impractical to consult managers each time. It is very easy to judge the wisdom of operators' decisions afterwards, but difficult to give them guidance beforehand.

\section{Issues and problem areas}

In these ambiguous situations, operators use tacit knowledge to make decisions about when to leave the plant alone, when to intervene, and how to intervene. There is a need for further research into how to better support operators to make these decisions in those complex situations where judgement is required. Thus, it is important to better understand the situation in which operators are required to apply their judgement to a production process, and this is our area of interest.

\section{Methodology}

\section{Research question}

In this work, we were specifically interested in finding how to differentiate between operator activities that are tampering vs. those that are valid applications of tacit knowledge.

- In managing complex manufacturing operations, what situations require manual intervention? Why? What causes the need for manual intervention?

- How are decisions made with manual intervention? How do operators react to the changes in process variables? Does this depend on the operator and his/her experience and knowledge (tacit knowledge)? How 
consistent are the manual interventions? In a given situation, do two operators make similar decisions?

- How effective is the manual intervention? Is it helping or hindering the output variables (quality, yield, etc.)? Under what conditions does this occur? What is the financial impact of poor practices of manual intervention?

- What are the best practices of manual interventions?

- Where in a complex process is the variability occurring?

Our primary purpose was to determine where in a given production process the tacit knowledge is making the biggest effect on outcomes. We accept that practical production plants only measure a small fraction of the many variables available, and that there are many operator actions that are not controlled by standard operating procedures or control charts. The problem is to find, from out of this vast set, which are the variables and tacit operator actions that are most affecting output quality.

\section{Context}

The specific case under consideration was a poultry rendering plant. Issues with the plant were the variability in output qualities, despite the plant being largely automated and operated by a supervisory control and data acquisition system (SCADA). The variations were not major, but were perplexing nonetheless. The plant managers had identified that there were differences in outputs between shifts and were beginning to wonder whether hitherto unidentified actions of operators were making the difference.

\section{Approach}

There were three components to our approach, undertaken in parallel. The one work stream was to construct a process model of the plant, identifying the operational activities and the known variables. A system engineering approach was taken to develop a functional representation of the plant. We were particularly focussed on ensuring that all the known SCADA and statistical variables were represented and also the potential operator judgements. The flow charts that are commonly used in the production environment are inadequate for this task, since they only represent the explicit component of the workflow, and are too simplistic. Consequently, we used the integration definition zero (IDEF0) notation (FIPS 1993) to represent this process model, since this better represents detail.

A second and concurrent work stream was to apply statistical methods to analyse the process variability of the plant. The data were provided by the plant managers and represented the main process variables and outputs comprising several months of operations. Statistical tools that were used included ANOVA and feature selection, within 'Statistica' software. The analysis identified which categorical or situational variables were most affecting the variability of output quality. We then used this information to guide the search for operator judgement activities in relevant areas.

A third set of activities were the knowledge management processes of identifying where tacit knowledge occurs within the overall workflow. This involved fieldwork: personal interactions with operators. We approached this by asking operators, 'Where in this process do you need to apply your own judgement?', and then, 'How do you make that decision?' We found operator judgement to be a more accessible concept for operators than the more abstract idea of tacit knowledge. Also, a deliberate part of the approach, we did not invite operators to a formal meeting to extract their knowledge, which might contribute to their unease, but rather embedded a researcher in the plant thereby permitting more natural conversations. To achieve this, a research engineer spent time in the plant, talking to the engineers, managers, and supervisors, and examining the SCADA system. It possibly helped our method that we used an engineer for this, but since we did not vary this part of the intervention, we cannot comment further. We sought to find out where operator intervention and judgement decision-making was occurring. We were guided in this search by the statistical analysis, which identified certain variables identified as being the main contributors to variability in quality. We identified, in discussion with supervisors, the operators with greater or lesser experience with the plant, and termed these 'expert' and 'novice' operators. These operators' actions were kept separate during the analysis. We then overlaid these operator judgements on the process model (see first work stream).

The method therefore involved the conjoint application of systems engineering, statistics, and knowledge management principles, in the context of a case study.

\section{Results}

\section{Context}

The rendering plant recycles the waste from the main plant which produces poultry meat for human consumption. The nature of the waste includes meat, carcass and bone, feathers, offal, blood, and birds that are dead on arrival. The waste is processed into a protein-rich dry meal and separate liquid oil (or tallow). Both these products are sold to other manufacturers as an ingredient for animal feeds. To achieve these outputs, the waste is cooked at a high temperature and pressure, while being agitated. Thermal 
processing is required to remove all vegetative microorganisms and ensure inactive chemical substances are destroyed, as these are potentially harmful if consumed by animals. This is a critical control point in the process. The pressure assists to obtain that temperature. The agitation is achieved by a slowly rotating beater. Water is vented during the cooking process, and the product becomes drier. Thereafter, the product is discharged and fed via augers to a press where the oil is separated from the meal. The cooking and subsequent processes are a batch, as opposed to continuous, process. Each batch takes about 6-h talk time, and there can be up to three batches running somewhat concurrently. The firm's own process flow chart is shown in Fig. 1, and this was the starting representation for understanding the context and developing our subsequent more detailed representation.

The main plant output variables are ASH, FAT, MOISTURE, and PROTEIN. These are measured at the final output of the dry meal and involve laboratory samples. These measurements are done for each batch and are the primary measurements of output quality. The main variable of economic value is the PROTEIN, and thus, a high value is preferable. Residual FAT and MOISTURE in the meal are unwanted, and low values are therefore desirable: FAT should have instead been extracted as oil and sold separately where it has more value than in the meal, and MOISTURE is a non-value added substance that should have been evaporated off in the cooking process. The ASH represents all the other solid components of the input stream not already represented in the other variables and should be minimised as it has no commercial value. The term ASH arises because the laboratory method determines the residual solid content by pyrolytic methods. Ash is not deliberately added to the product.

The only reported variables for which there were historical data were PRESS PRESSURE, AUGER SPEED,

\section{PROCESS FLOW PROTEIN RECOVERY PLANT}

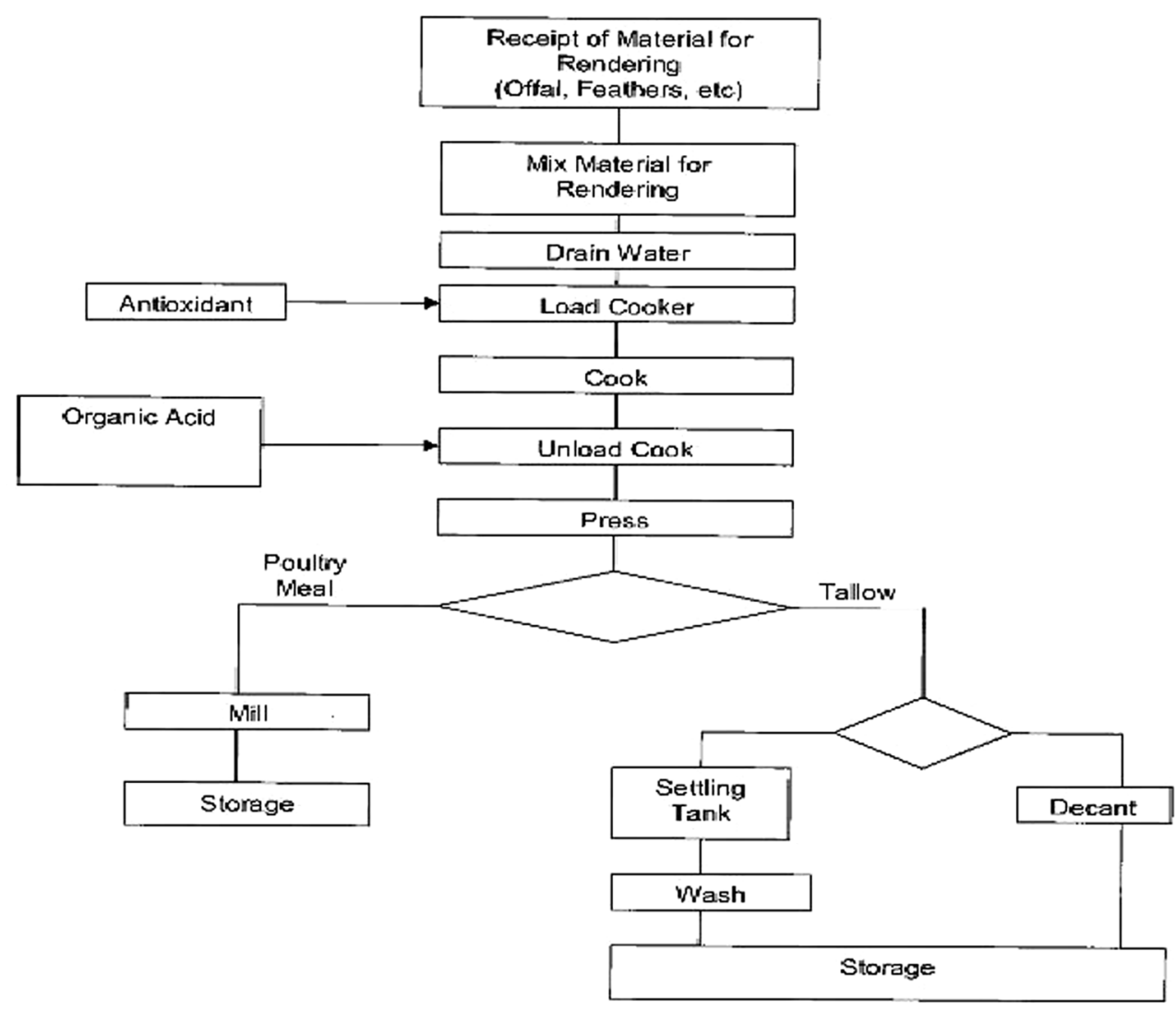

Fig. 1 Original flow chart of protein recovery process. This is the industry's own plant diagram 


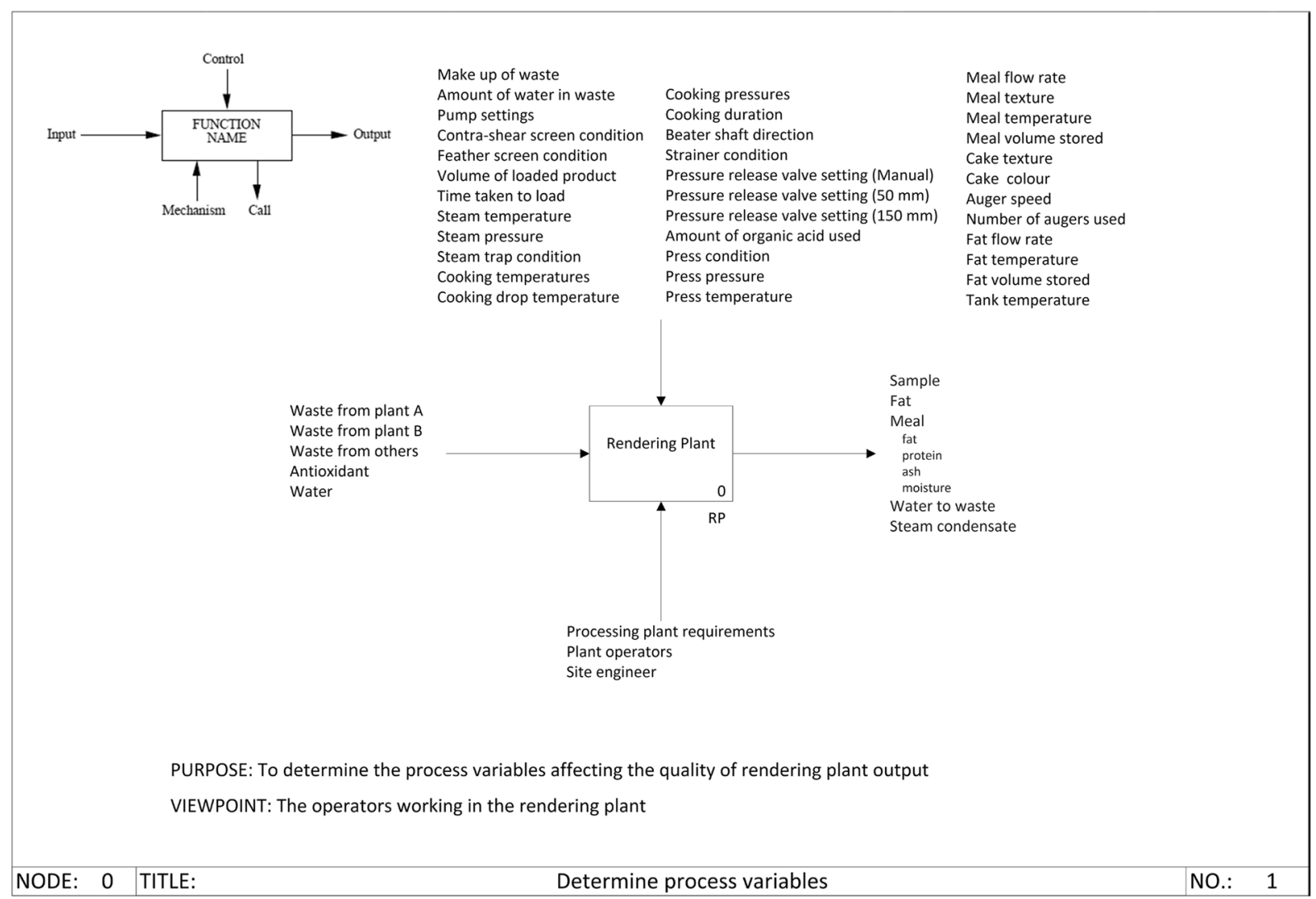

Fig. 2 Top level representation of the protein recovery plant: an overview of variables

and DROP TEMPERATURE. There are many other variables monitored in real time in the SCADA system, but these were not available as they were not recorded by the plant. Obviously, if our method was applied to a green-field plant, it would be advantageous to record as many of the variables as possible. However, the situation encountered in this case study is typical of many industries, where only certain key variables are recorded, and being able to use the limited data available is a realistic test of the method.

\section{Plant model in IDEF0}

The modelling method uses a structured, deductive process to decompose the process being analysed into multiple subactivities (functions) and for each deduce the initiating events, the controls that determine the extent of the outputs, the inputs required, the process mechanisms that permit the action to occur, and the outputs. It should be noted that this is a more detailed analysis than normally provided in production flowcharts, but, nonetheless, most of this information is readily available. The end result is a graphical model that describes the relationships between variables, thereby providing a synthesis of what is known about the causality whereby mechanisms convert the inputs into the outputs. The model is expressed as a series of flowcharts using the integration definition zero (IDEF0) notation (FIPS 1993; KBSI 2000). With IDEF0, the object types are inputs, controls, outputs, and mechanisms (ICOM) and are distinguished by placement relative to the box, with inputs always entering on the left, controls above, outputs on the right, and mechanisms below.

The IDEF0 model is hierarchical, with the top level being shown in Fig. 2. There is not a large amount of detail at this level, other than an identification of the main explicit variables. These are primarily the variables monitored by the SCADA system, and those were statistics reported for management purposes.

Greater detail becomes evident in deeper levels, and the diagram numbering system represents this hierarchy. The three main sub-processes to rendering are receiving, cooking, and processing (see Fig. 3). Each of these may have further sub-processes. In the case of receiving, there is no further detailed model available, but there are models for cooking (see Fig. 4) and processing (see Fig. 5).

\section{Statistical insights}

The main plant output variables are ASH, FAT, MOISTURE, PROTEIN, and the main process control variables are PRESS PRESSURE, AUGER SPEED, and DROP 


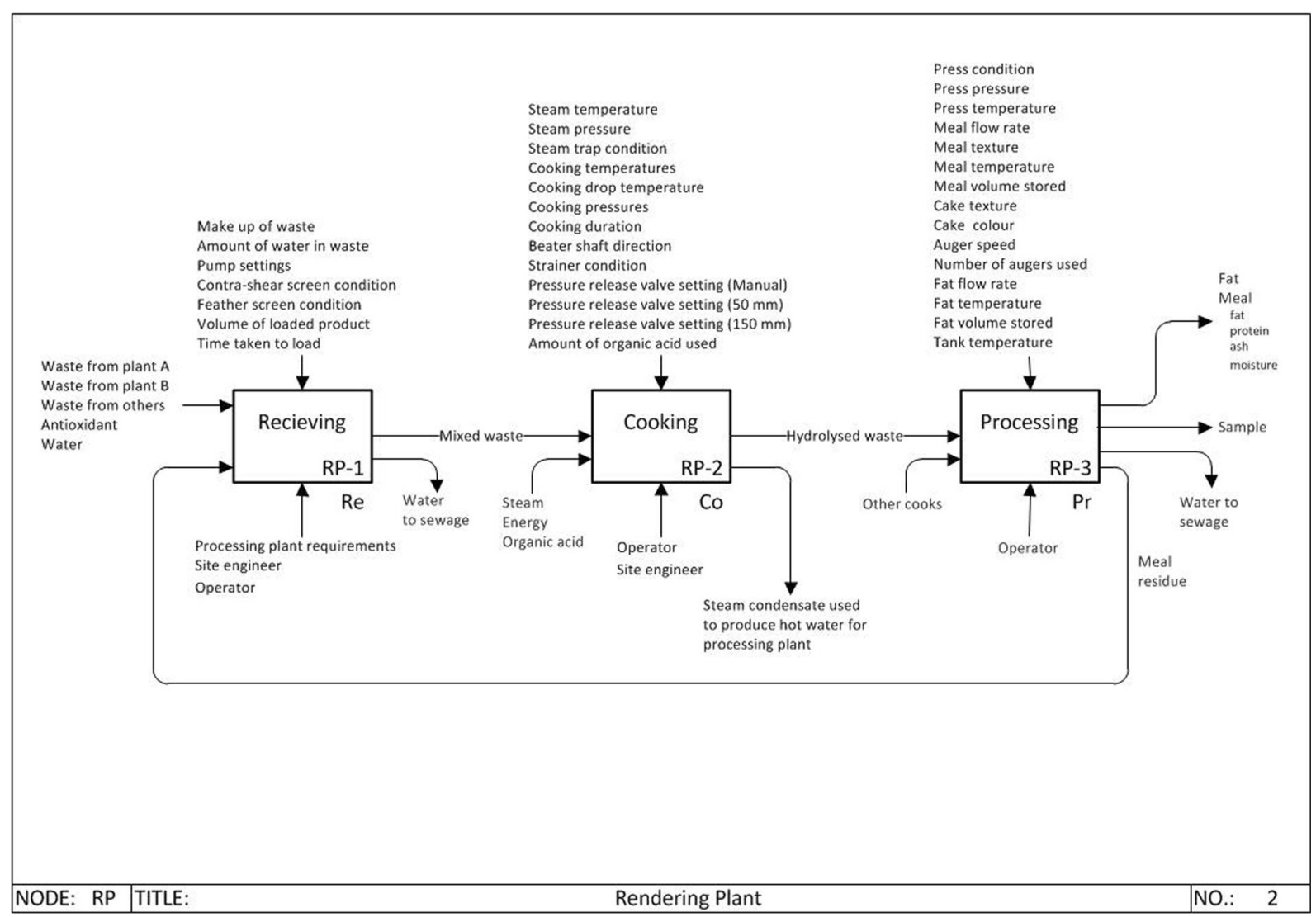

Fig. 3 Rendering process broken down by function

TEMPERATURE. A typical approach to this type of situation is to look at the control charts, and we explore this perspective first. Thereafter, we applied ANOVA and feature selection analyses.

\section{Control chart analysis of fat}

Taking FAT as an example, the question is whether the FAT content is statistically in-control. If it is control, manual intervention is not expected to be required, at least with reference to this variable. If out-of-control, one possible reason is that manual control is affecting the process quality, though there may be other reasons for this. The Xbar charts of sample mean and standard deviation were computed for a sample of the data and are shown in Fig. 6.

Explanations for these results were solicited from the operational staff. High value on date $14 / 6$ was ascribed to operator error. However, no explanations could be provided for the other out-of control situations. This seems a consequence of the long time delay between completing the batch processes, and the availability of the laboratory tests. Thus, the feedback loop to operators is not fast and perhaps also not fully effective. This leads to a recommendation to provide more immediate feedback to operators via run/control charts.

\section{ANOVA categorisation}

The ANOVA results ('Appendix A') show that there are significant differences between the COOKERS, which could depend on their physical construction (no two pieces of plant are identical in behaviour even if their construction is nominally identical), or how they are handled by the operators. Likewise, SHIFT differences exist, which could depend on the nature of the input material received (no data available on that variable), or personnel skill, or again how the operators treat the plant. These variations could not be explained by the operational staff. Taken together, these results suggest differences in the way operators interact with the plant.

\section{Feature selection}

With only a few recorded variables, it is feasible to conduct an exploratory ANOVA analysis, as shown above. However, there are two situations where this may be insufficient. The first is that some important process variables (e.g. PRESS PRESSURE) are not categorical variables and therefore more difficult to include as independent variables. The second is that a brute-force ANOVA approach is inefficient when there are many variables. Fortunately, there 


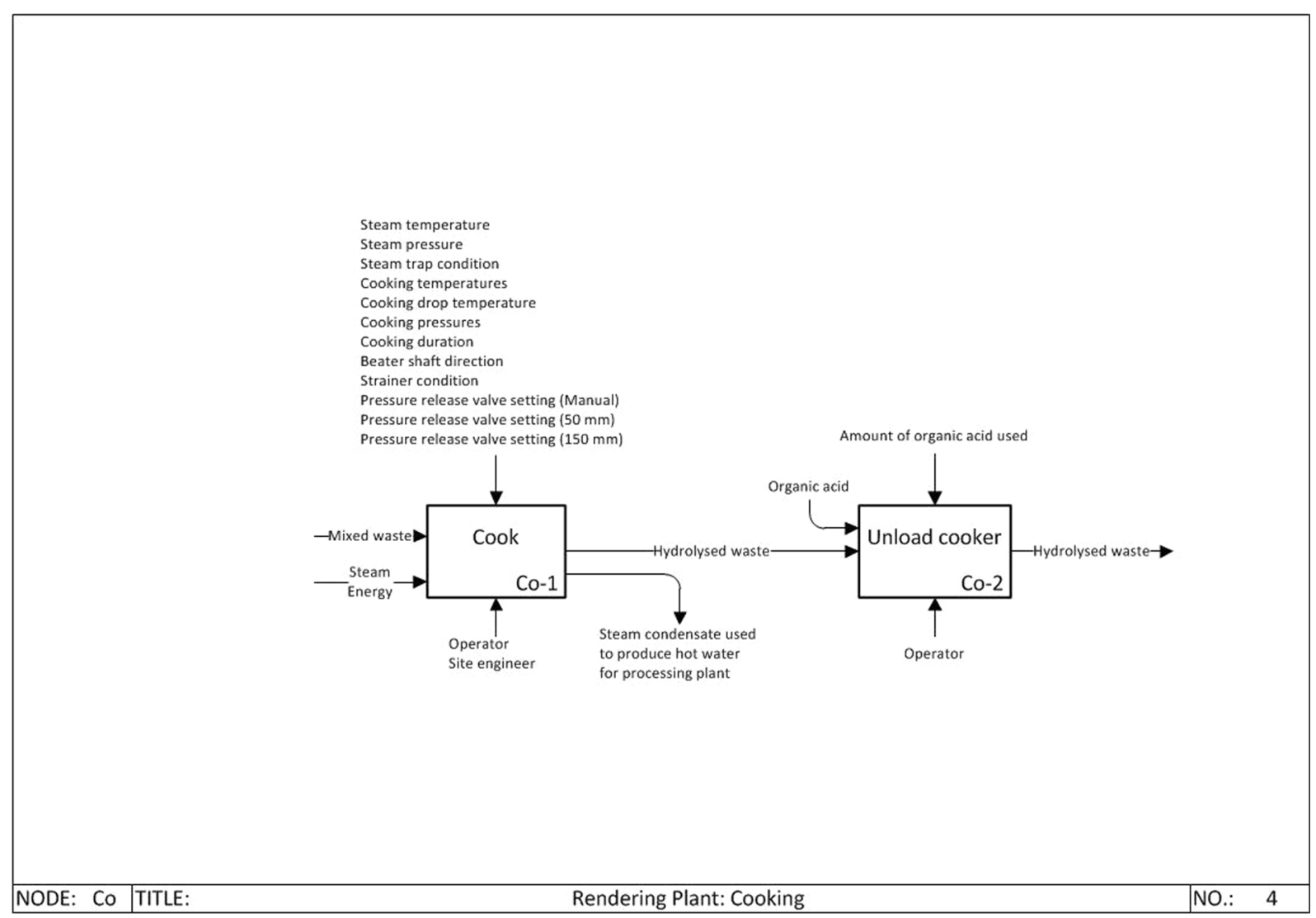

Fig. 4 Cooking process

are other statistical methods that can be used, such as feature analysis, as shown here. In this analysis, we were interested in which of the controllable variables (PRESS PRESSURE, AUGER SPEED, and DROP TEMPERATURE) were most important in the output variables (ASH, FAT, MOISTURE, and PROTEIN), which is to say, how much variability in those output variables is explained by the controllable variables. In considering these results, it is worth noting that there might be other unknown independent variables that are controlling the outputs. The results of this analysis are shown in 'Appendix B'.

The results show that:

- ASH depends mainly on SHIFT and AUGER SPEED.

- FAT depends mainly on PRESS PRESSURE, AUGER SPEED, and COOKER.

- MOISTURE depends mainly on COOKER.

- PROTEIN depends mainly on PRESS PRESSURE.

Taken together, we interpreted these results as suggesting that AUGER SPEED and PRESS PRESSURE were important. We therefore directed our fieldwork to enquire about operator judgements in these areas.

The feature selection shows that residual FAT in meal is associated with PRESS PRESSURE, AUGER SPEED, and COOKER. To find the direction of association, we selected the two top most important variables and produced the response surface based on a polynomial, see Fig. 7. Functions other than polynomial could have been selected to fit the data, but they all produced much the same trends. In any case, there is not necessarily any information in the equation itself, i.e. there is no reason to believe that the underlying process mechanics follow a polynomial (or other) relationship. Instead, it is the insights that can be extracted from the trends, and particularly, the practical implications for the operators that are valuable.

We note this is not fully populated with data. Nonetheless, to the extent to which it accurately represents the plant behaviour, it predicts that low FAT is more readily obtained with high PRESS PRESSURE and low AUGER SPEED. It is understandable that high PRESS PRESSURE should squeeze out more oil, hence low FAT. However, the mechanics whereby low AUGER SPEED could be causally connected to low FAT is non-intuitive, and we return to this later.

\section{Link analysis}

As a final exploratory tool, we apply link analysis. This is a data mining method that is used to seek common occurrences and associations between variables. It is also a 


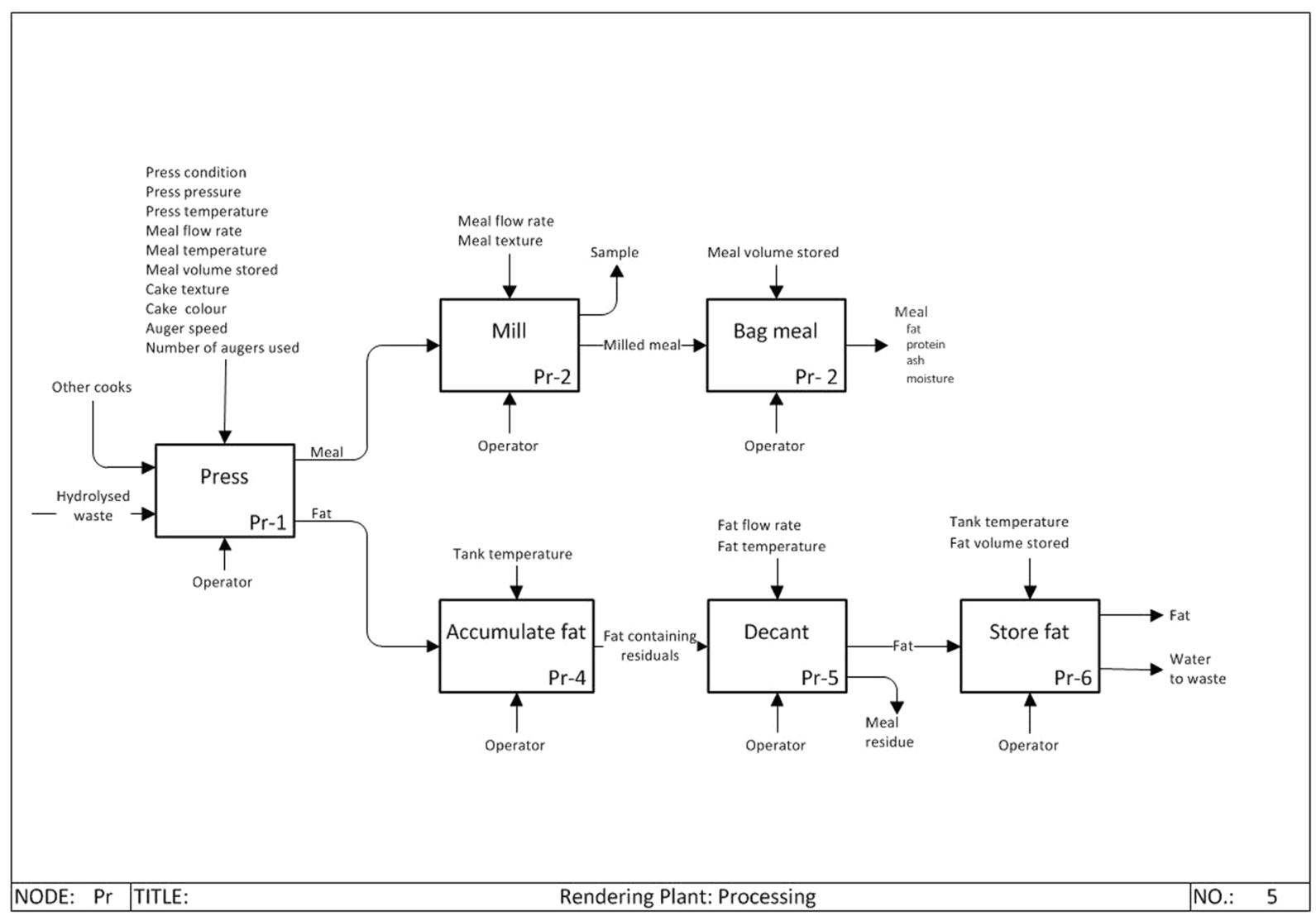

Fig. 5 Processing process

Xbar-S Chart of Fat
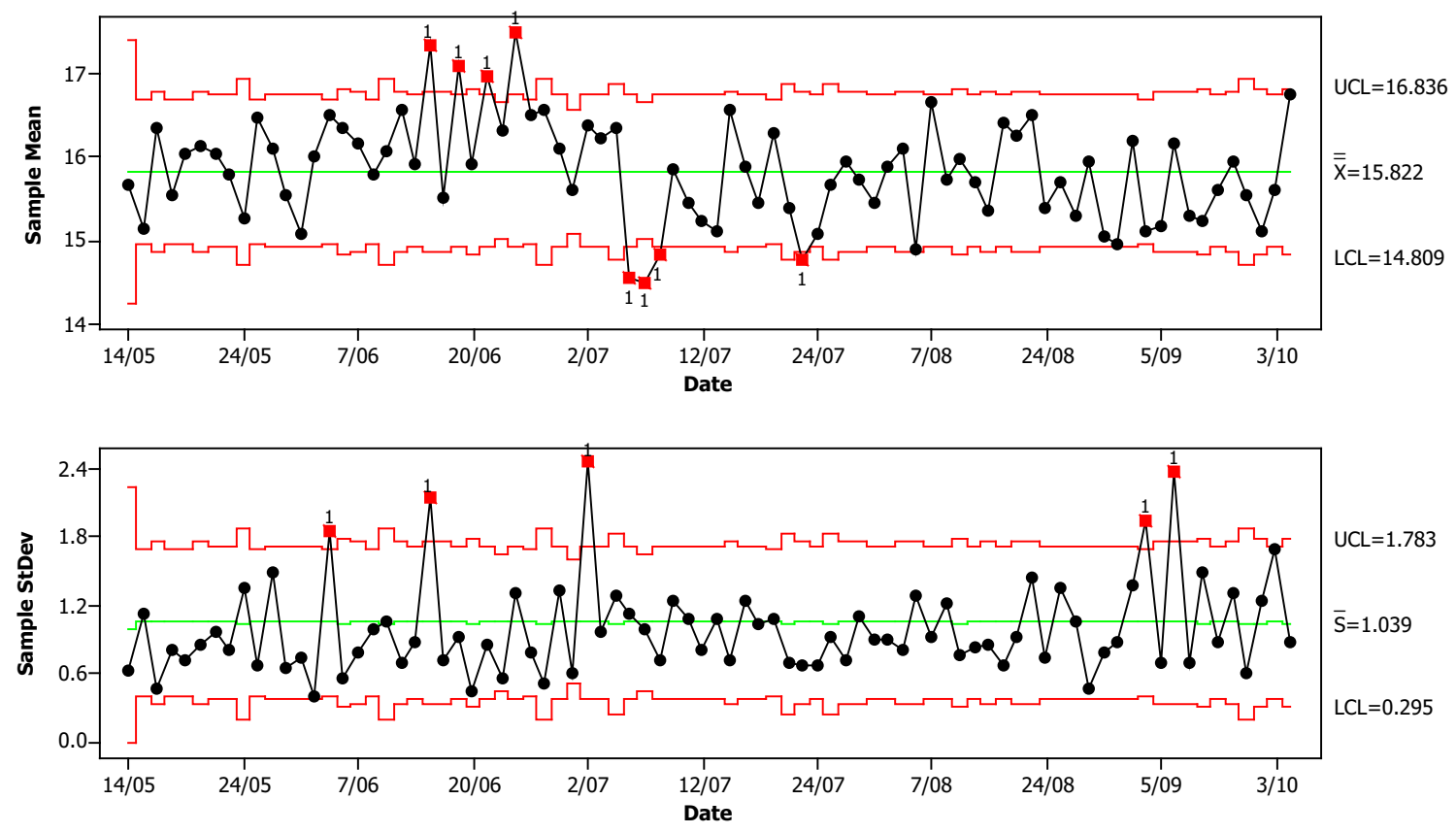

Tests performed with unequal sample sizes

Fig. 6 Rendering plant control chart analysis 
3D Surface Plot of Fat against Press Prsure and Auger Speed Sheet1 in ShiftOutputs.stw $12 v^{*} 941 \mathrm{c}$

Fat $=52.8345-0.5755^{*} x-12.0015^{*} y+0.002^{*} x^{*} x+0.1055^{\star} x^{*} y+0.8351^{*} y^{*} y$

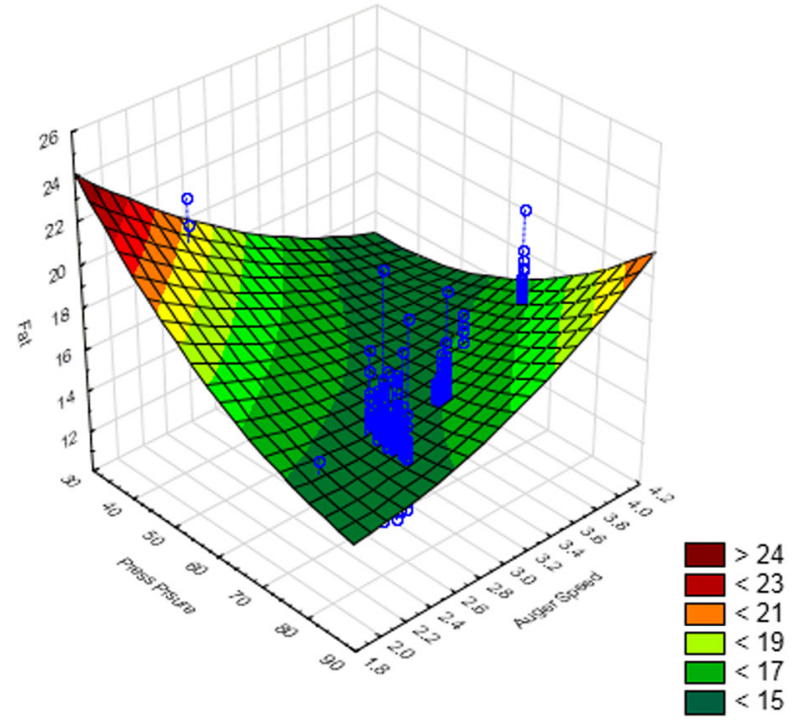

Fig. 7 Response surface for residual FAT. Low values of FAT are preferable

posterior method and does not require any prior assumptions about causal relationships between variables, unlike the a priori methods. It is therefore suitable for seeking any associations that might have been missed, and in this regard, can be considered a useful de-biasing method. The method is also suitable for large datasets, and even more efficient (in analyst's time) than the feature selection method, since it looks for all associations at once. Also it can, with careful use, accommodate continuous and categorical variables. The results are shown in Fig. 8. In this particular case, there are no great insights obtained other than some observations about PROTEIN that are consistent with earlier results. Nonetheless, we include this tool in our overall method because it would be the most suitable of all three statistical methods for very large data sets (which can easily be generated by SCADA systems).

\section{Engineering insights}

Interpreting the statistical results is greatly assisted by having an engineering and operational knowledge of the plant, which is represented in the plant model. There is a flow of material through the plant, and this imposes a time dimension and a cause-and-effect result on quality outcomes. This helps identify the plausibility or otherwise of certain associations. Consequently, if there is a variable that is measured downstream of a particular process, then it is reasonable to be sceptical about the possibility of that variable affecting the upstream process (obviously there are exceptions and common causes are possible).

\section{Operator judgement}

The statistical analysis and plant modelling processes were conducted concurrently and mutually informed each other. Importantly, the statistical analysis also identified which variables were associated with variation in the outputs, and the plant model showed where those variables arose. The value of this is the ability to direct fieldwork at the area where the variability is occurring, specifically to seek for operator judgement activities.

In this particular case, the statistical analyses identified that the processes around the COOKER and PRESS were of particular interest. We therefore directed fieldwork at finding out what the operators were doing at these stages. We were interested in identifying where the tacit knowledge was being applied, and the nature of that knowledge, hence the questions like, 'Where in this process do you need to apply your own judgement?' The results are differentiated by operator experience (expert vs. novice). This operator's responses were represented as constraints on the plant model diagrams, for the COOK and PRESS operations. The results revealed some interesting new insights into the plant.

\section{Cooker: operator judgements}

The critical judgement, according to operators, is when to drop the pressure on the COOKER, see Fig. 9. This temperature determines the amount of residual water in the meal (hence MOISTURE) and the extent to which the protein is denatured (hence PROTEIN). However, this did not show up as the most important variable in the statistical analysis: instead, COOKER was more important. The reason becomes clear after discussion with operators, and concerns a relationship between the DROP TEMPERATURE and a maintenance task. It turns out that one of the more unpleasant tasks is cleaning the strainer in the pressure release valve on the cooker: the pressure release valve is awkwardly positioned and hence difficult to service. The organic material entrained in the steam clogs-up the strainer when the cooker is overloaded (batch size too large in pursuit of productivity). If the strainer is not cleaned after it has been clogged-up during an overloaded cook, then the pressure release valve does not work properly during the next cooking cycle. A faulty pressure release valve can lead to excessive pressure build-up in the cooker which in turn can lead to the cooker exploding. The problem is combated by installing a weighing system for cooker loading. 


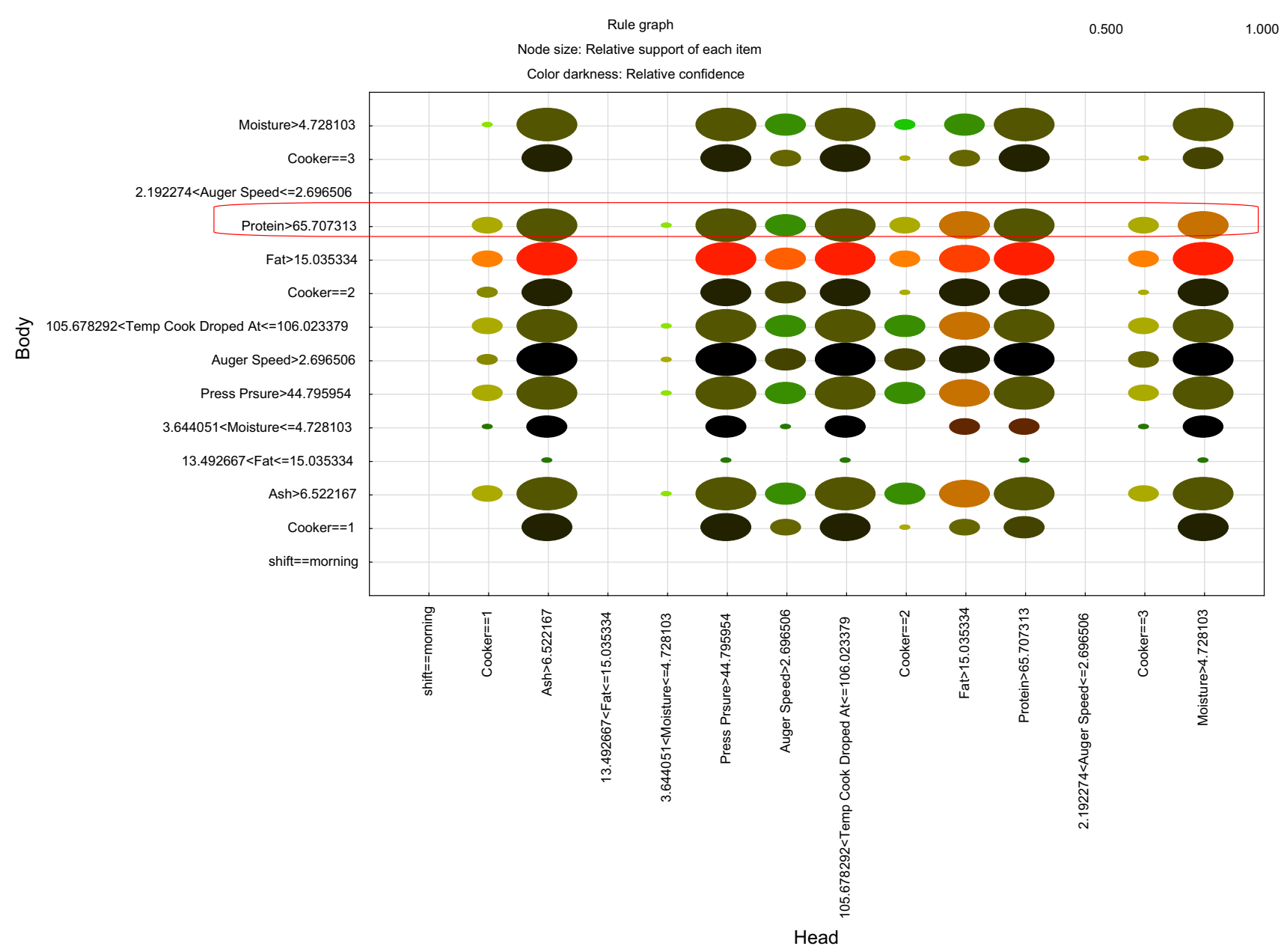

Fig. 8 Link analysis (min support 0.40, min confidence 0.4, continuous variables reduced to three levels, maximum three items in an item set). The results for PROTEIN are highlighted, and these

\section{Press: operator judgements}

The press is where the oil (and water) is extracted from the cake. Recall that the statistical analysis had shown that high PRESS PRESSURE and low AUGER SPEED were the best operating characteristics. The importance of high PRESS PRESSURE is entirely plausible, but the requirement for low AUGER SPEED is not so intuitive, and could not at first be explained by operators. The AUGER SPEED variable partly measures the rate of transfer of material from the cooker to the press.

Discussion with operators showed that experts and novices were doing slightly different things here. The task involves selecting to operate from one to three augers, the speeds thereof, and manually shovelling material as necessary, see Fig. 10. The experts identified only two variables as important, the number of augers and the speed thereof, and were seeking to maintain a steady flow into the press. By comparison, novices were attempting to make a suggest that high PROTEIN is associated with high ASH, high PRESS PRESSURE, and a number of other variables that may be unimportant

more complex judgement on additional variables including the colour and texture of the cake, and actively looking at press characteristics. The poorer results of the novices suggest they were unnecessarily overworking the decision.

The fieldwork revealed interesting features of the mental models of the two classes of operators. High AUGER SPEED would seem more desirable for productivity considerations, and indeed, this was a primary consideration for novices. They felt more productive feeding the material through faster. Possibly, it also meant they could get this job done quicker and return to the more comfortable environment of the control room. However, the expert operators had discovered, rather counter-intuitively, that low AUGER SPEED produced better results. With some engineering knowledge of the plant and in discussion with operators, it was possible to identify the mechanical explanations for the effect. We found that the mass flow capacities of the augers exceeded that of the press (which of itself is not a fault), so that operators were able to, 


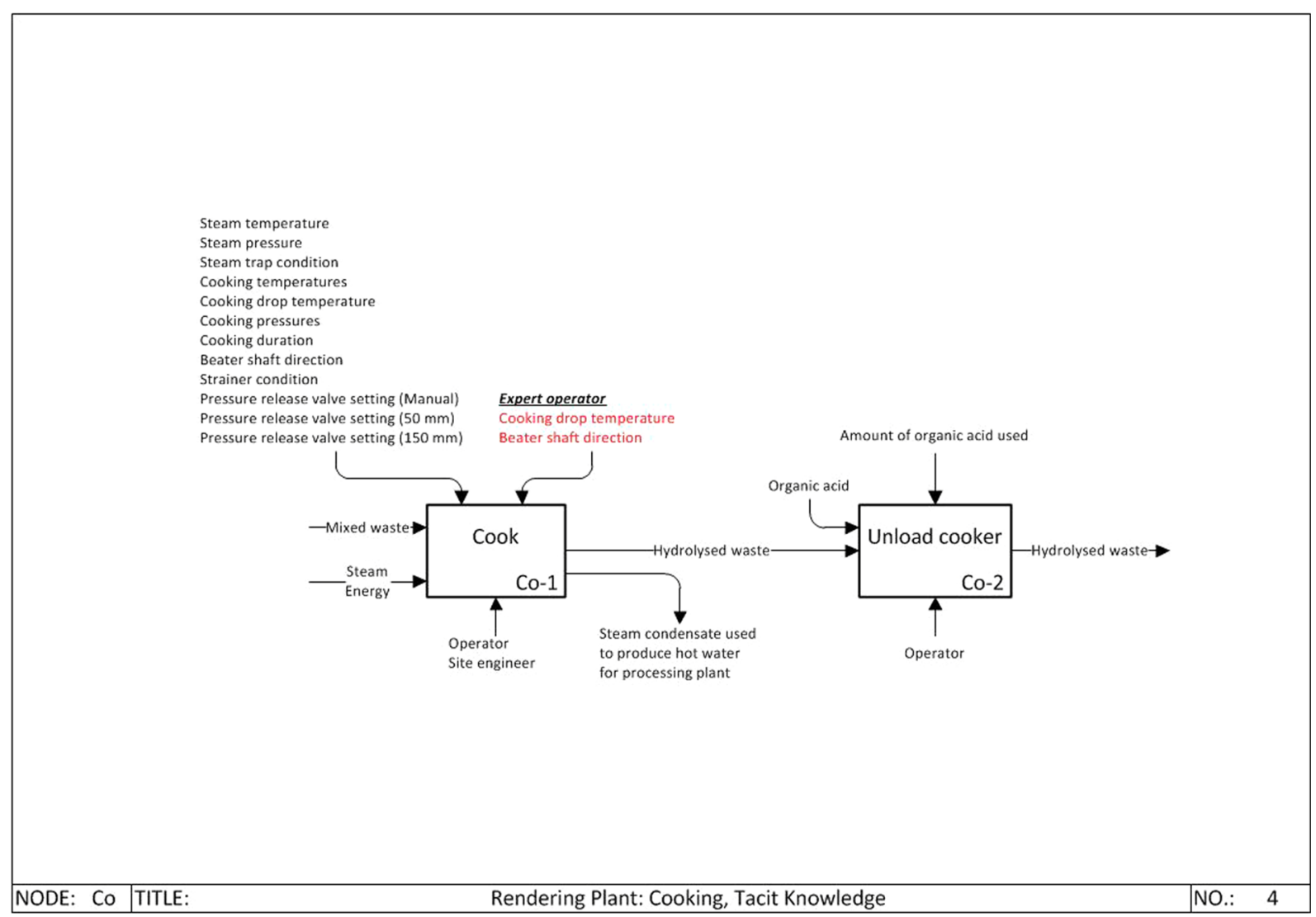

Fig. 9 Cooking process with identified tacit knowledge variables

inadvertently and with productivity in mind, set the auger speed to offer too much material to the press, thus decreasing the press efficiency and causing lower oil and water extraction.

Clearly, there are some implications for this particular plant, but more importantly what this shows is the importance of operators' mental models of the plant. These models develop with experience and hence time. Novice operators appear to have mental models that are strongly influenced by productivity imperatives: they believe they are doing the right thing by working the plant faster. They do not initially understand the plant well enough to be able to anticipate the unintended consequences. That knowledge only occurs over time. It is also relevant to note that it may be difficult for plant managers to anticipate the intricacies of the plant behaviour, since they are removed from that level of operational detail.

We summarise the results by representing the expert and novice behaviours on the IDEF0 functional chart, see Fig. 10. This captures the tacit knowledge of operators and represents it in a way that can be used for further training and improvement cycles. Note that we have only done this for the PRESS activity. The reason is that the statistical analysis showed that this was the area that contributed most to the variability in quality. This is consistent with the usual quality improvement processes that seek to eliminate the worst offenders first and then look at the other processes subsequently.

A positive feature of the method is that it readily permits concrete implications to be extracted for practitioners. In this case, the specific recommendations for the plant operators and managers encompass some immediate as well as long-term improvements, see Appendix $\mathrm{C}$ by way of example.

\section{Discussion}

\section{What has been achieved?}

This paper makes several novel contributions. The first is the methodological contribution of developing a method to determine where in a given production process the tacit knowledge is affecting the quality outcomes. We showed how a combined usage of systems engineering, statistical analysis (ANOVA, feature selection, and link analysis), and knowledge management methods (elucidation of tacit knowledge by targeted fieldwork) could be used to identify 


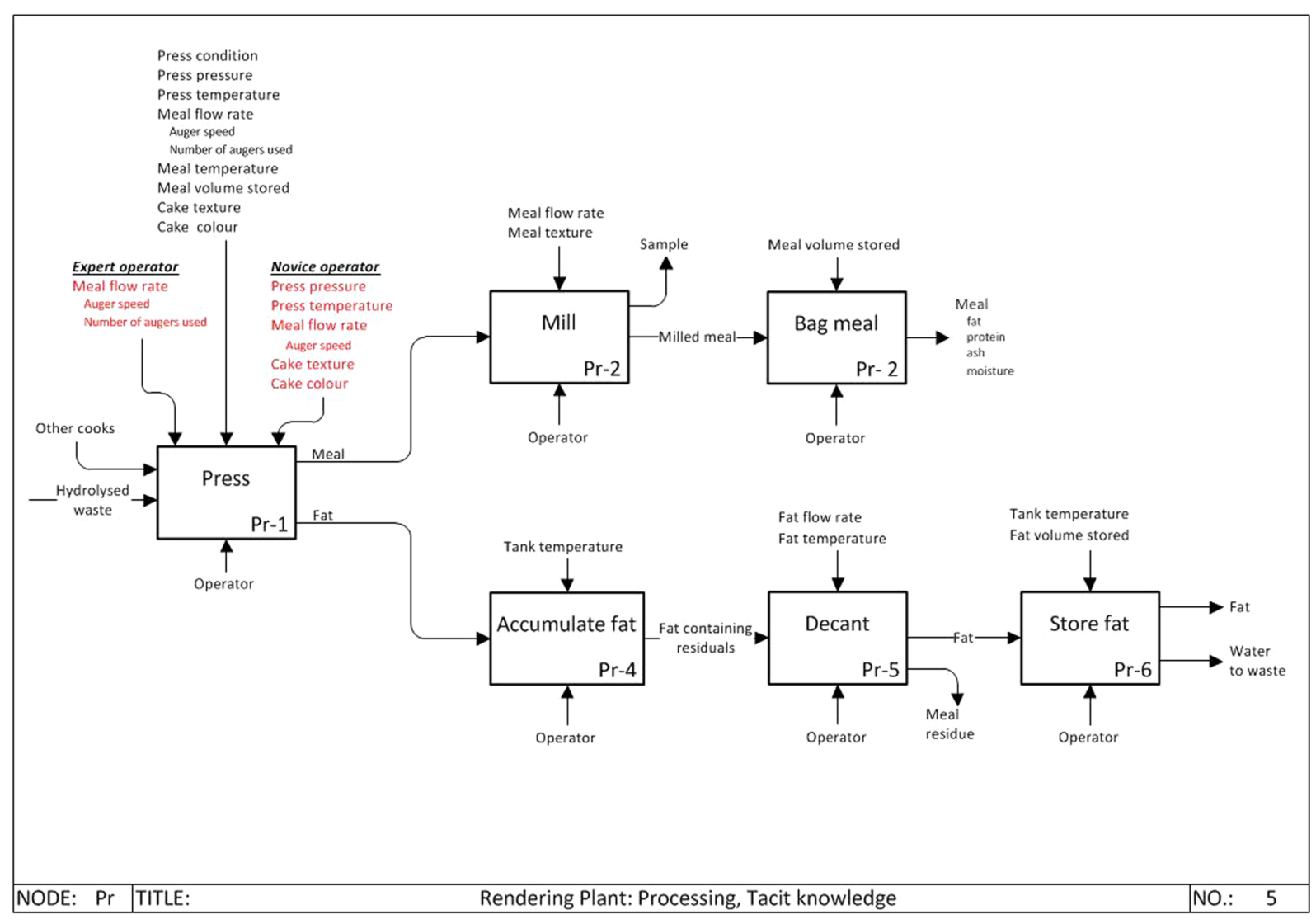

Fig. 10 Press process with identified tacit knowledge variables

where quality-critical operator judgements were occurring, where these had previously been hidden to the operators and managers. This integrated approach has not been demonstrated before.

The system engineering contribution was the development of the plant model, the provision of production insights to inform the interpretation of the statistics, and the representation of the operator judgements. Suitable mechanisms for the statistical analysis were identified as control charts, ANOVA, feature selection, and link analysis. It was shown how these could, singly or collectively, be used to identify areas in which to prospect for operator judgement activities. Importantly, the suite of statistical tools includes some, like link analysis, that may be used for large data sets. The method was demonstrated by application to a case study.

A second contribution is the provision of a novel methodology for knowledge management. The usual KM method involves codifying the tacit knowledge, storing it (typically in a computer database), and then (somehow) making it available to other users. However, all steps in such KM systems have proved to be highly difficult to implement in practice, with barriers appearing at each of those stages, especially the first. We suggest this is because such approaches to KM are too intrusive and motivationally conflicting for operators. We have developed a different method of KM, specifically for the production engineering environment, that is designed to avoid these barriers. Our method is not to even attempt to extract the actual tacit knowledge of operators, but rather to identify where the tacit knowledge was being applied, and the nature of that knowledge. The style of questions we use are non-judgemental questions and nor do they require the operator to divulge his cognitive algorithm (which he may not be able to do, or want to express). Our questions are: 'Where in this process do you need to apply your own judgement?', and then, 'How do you make that decision?' or 'What factors do you have to take into account when making this decision?', and similar conversational questions.

Our method of $\mathrm{KM}$ is therefore designed with continuous quality improvement in mind: it identifies where the variability in human action is occurring, and then leaves it to the operators to make the improvements through the usual quality circles or kaizen improvement events. From there, they can make SOPs or set up control charts as they see best: our method is empowering rather than prescriptive about what they do next. At the same 
time, it can result in specific recommendations for operational improvements, as we have demonstrated, so it is not merely an abstract method. And while conventional KM documents its knowledge in databases, our method expresses the knowledge back on a representation which is easy for production people to comprehend: on a production flow chart. Ours is therefore a very different approach to the otherwise top-down adversarial method of KM.

A third contribution is that we have developed a method whereby operator judgement may be included in the continuous quality improvement processes. This has not been demonstrated before. Instead, the existing improvement processes are strongly focussed around the Six Sigma method which involves collecting data, analysing it, and improving the production system. Our contribution is to have piloted a method whereby operator judgement can be included in the data collection process. We have shown how standard statistical tools including control charts, ANOVA, feature analysis, and link analysis (data mining) may be used to identify likely areas where operator judgement occurs, and we have provided a method whereby the relevant questions can be asked of operators in a non-threatening manner.

A fourth contribution was the identification of some interesting attributes of the mental models of expert as contrasted with novice operators. In particular, it was identified that novices were focussed on meeting throughput objectives, and their incomplete understanding of the plant characteristics lead them to inadvertently sacrifice quality in the pursuit of productivity in certain situations.

\section{Implications}

Several novel propositions emerge from this line of thinking. We suggest that the idea that tampering is bad is too simplistic. Real production plants are complex places, and it is naïve to assume that all the key variables can be identified and controlled, and that all such controlled processes are stable. Also, the whole tampering paradigm is premised on the idea that it is only stochastic machine variability that is important, and this is underscored by Deming's use of a very passive machine, the funnel. Left to itself with marbles of a given size, a funnel will simply do one thing: it has only one operating state, and the stochastic variability is only around that one state. The tampering way of thinking ignores the possibility, which we show is real, that a plant can have multiple operating states and variable input feed material, and therefore needs active and intelligent management through those transitions. While we acknowledge that in many cases intelligence may be provided by electronic and software systems, viz. the SCADA system evident in our case study, the practical reality is that human operators are still required. Production environments do require operators to act with personal agency, and that necessarily means an element of judgement in deciding whether or not, or even how, to intervene. We therefore challenge the prevailing paradigm that states that tampering is bad: we suggest that construct is overly simplistic and relevant only to certain well-defined production situations. We also refute the funnel experiment as naïve and not representative of the complexity of real production environments. We suggest the real challenge is how to differentiate those situations where operator judgement is welcomed from those where it is not. The method we have developed here, combining systems engineering, statistical analysis, and knowledge management, offers a way to solve this problem.

\section{Limitations and opportunities for further research}

We have developed a method for identifying where operator judgement occurs within a production process, based on a plant model, statistical analysis of historical production data, and fieldwork. However, there are a number of limitations in our work. One of these is the subjectivity of the process, and associated with that the reliance on specific insights. The success of the method may depend on the knowledge and skills of the investigators. Thus, replication of the method would be an interesting future research topic. Another limitation is that the work was only completed to the diagnostic level. The scope excluded monitoring the results of changes. Consequently, there is no reporting of the ultimate efficacy of the method. It would be interesting to apply the method to a longitudinal study.

\section{Conclusions}

The purpose of this work was to determine where in a given production process the tacit knowledge is making the biggest effect on outcomes. A novel methodology was developed to address this problem. We show how a combined usage of systems engineering, statistics, and knowledge management fieldwork may be used to identify where quality-critical operator judgements were occurring, where these had previously been hidden to the operators and managers. The systems engineering perspective provides a method to develop the plant model, provides the production insights to inform the interpretation of the statistics, and allows the operator judgements to be represented. The statistical analysis, in the form of control charts, ANOVA, feature selection, and link analysis, was used to identify the production variables most affecting variability in quality. In combination with the system model, this permitted 
fieldwork to be directed to specific areas to prospect for operator judgement activities. The results show that it is possible to identify where operator's tacit knowledge and judgement is contributing to quality outcomes. It achieves this without struggling with the usual codification barriers to knowledge management. The method is compatible with other continuous quality improvement methods. We conclude that tampering cannot be considered to be universally bad. Instead, operators' intervention is frequently a practical necessity of real production plants. The debate should not be about tampering vs. intervention, but how to differentiate those situations where operator judgement is appropriate from those where it is not. The method we have developed here, combining systems engineering, statistical analysis, and knowledge management, offers a way to solve this problem.

Author Contribution statement VP and DP provided project oversight. VP arranged the case study. PvG did the field work, and created the plant and operator model. VP undertook the control chart analysis. DP provided the statistical analyses and modelling methodology. PvG and DP provided the engineering interpretation. DP assembled and edited the paper.

Acknowledgments We acknowledge with gratitude the willingness of our industrial case study organisation, who wish to remain anonymous, for hosting the fieldwork.

Conflict of interest The authors declare that there are no conflict of interests regarding the publication of this article.
Open Access This article is distributed under the terms of the Creative Commons Attribution License which permits any use, distribution, and reproduction in any medium, provided the original author(s) and the source are credited.

\section{Appendix A: ANOVA statistical analyses}

The results below show the ANOVA decompositions categorised by SHIFT and COOKER, see Figs. 11, 12, 13, $14,15,16,17$ and 18. These analyses were conducted in a posterior fashion (as opposed to a priori hypothesis), and their purpose was exploratory: to identify the categories of variables that are associated with differences in output quality. Therefore, the dependent variables were the quality measures of PROTEIN, FAT, etc., and the categorical variables were SHIFT and COOKER.

\section{Appendix B: Feature selection statistical analysis}

In this analysis, we were interested in which of the controllable variables (PRESS PRESSURE, AUGER SPEED, and DROP TEMPERATURE) were most important in the output variables (ASH, FAT, MOISTURE, and PROTEIN), which is to say, how much variability in those output variables is explained by the controllable variables.

Feature selection identifies which controllable variables ('features') have the greatest importance in determining the
Fig. 11 ASH output from plant, represented as ANOVA decompositions categorised by SHIFT and COOKER. Low ash is preferable. The results show statistically significant differences, with most of variability arising in the SHIFTS rather than the COOKERS. The afternoon SHIFT shows the highest ASH, and while the magnitude of this is not great, the difference is statistically significant $(p<0.00000)$
Cooker*shift; LS Means

Current effect: $F(4,848)=1.0190, p=.39649$

Effective hypothesis decomposition

Vertical bars denote 0.95 confidence intervals

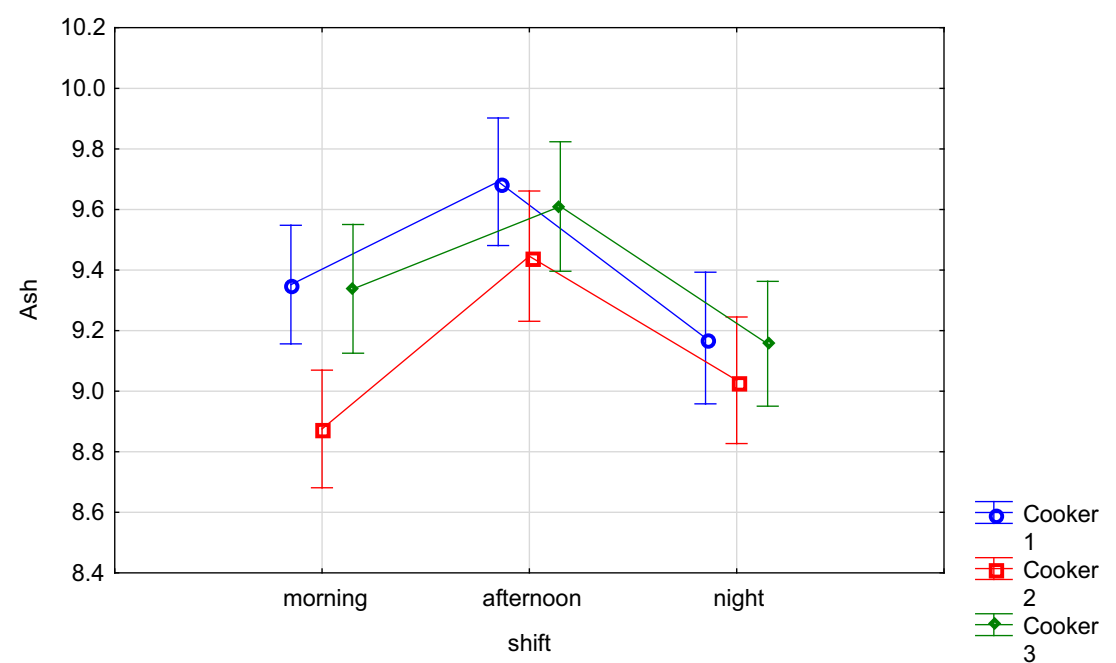


Fig. 12 FAT output being the residual fat in the meal from plant. Low fat is preferable. The COOKERS behave quite differently. Of the COOKERS, number 1 consistently produces lower FAT. There is also an interaction with SHIFT:

COOKER 2 produces less FAT on the afternoon SHIFT

Fig. 13 MOISTURE output from plant. Low moisture is preferable. The night SHIFT produces significantly $(p=0.031)$ more moisture than the other shifts
Cooker*shift; LS Means

Current effect: $F(4,848)=2.1892, p=.06843$

Effective hypothesis decomposition

Vertical bars denote 0.95 confidence intervals

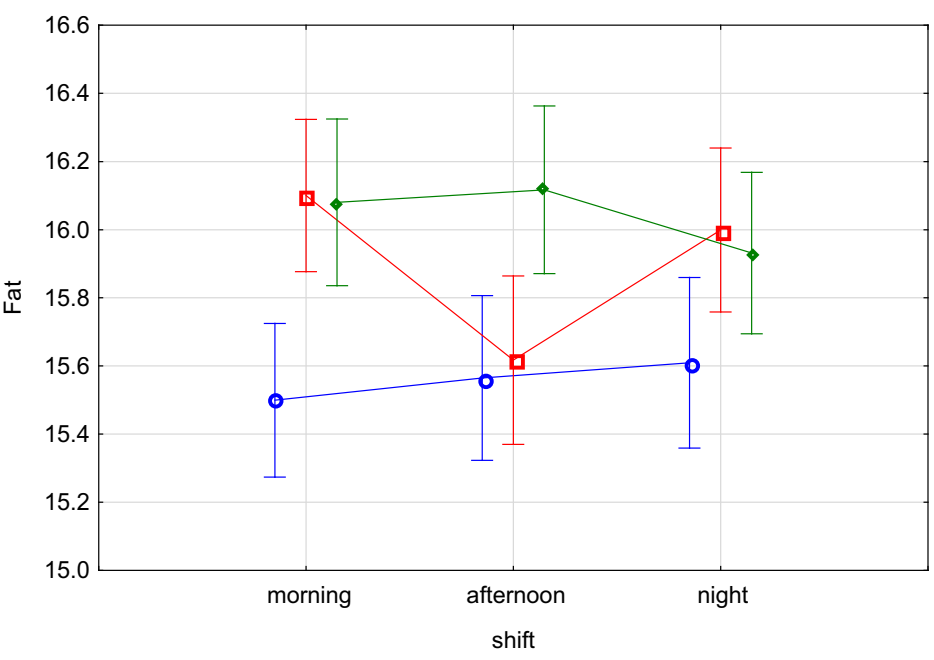

- Cooke 1 Cooker 2 Cooker

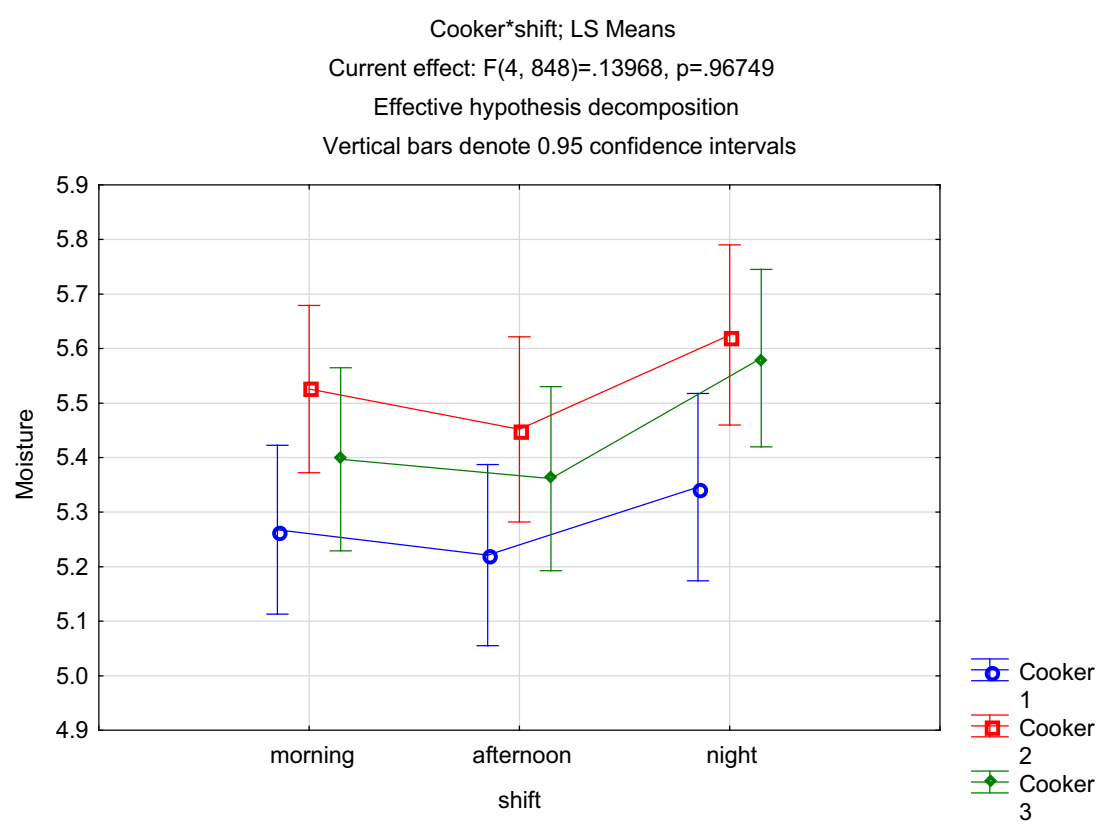

outcome variables of interest. The method is powerful as it makes no assumptions about the nature of the relationship between the variables, whether linear or any other. It also handles continuous and categorical variables. This is a form of predictive data mining. The method used was feature selection within the Statistica analysis tool. The resulting predictions are not so much for causality as association. The results are presented in Figs. 19, 20, 21 and 22. The variables with greater importance have larger F-values, shown here as longer bars.
The predictors obtained above were used to identify the variables that were worth exploring further in the fieldwork and for inclusion in the IDEF0 model building.

\section{Appendix C: Implications for plant managers and operators}

There is small magnitude but statistically significant differences in plant output as measured by ASH, FAT, MOISTURE, and PROTEIN. Several lines of action are recommended. 
Fig. 14 PROTEIN output from plant. High protein is preferable. The differences between the SHIFTS are not statistically significant, but the difference between the COOKERS is, with COOKER 1 producing a slight, but significant $(p=0.00095)$ more PROTEIN

Fig. 15 COOKER 1 produces a slight, but significant $(p=0.00095)$ more PROTEIN
Cooker*shift; LS Means

Current effect: $F(4,848)=.53448, p=.71044$

Effective hypothesis decomposition

Vertical bars denote 0.95 confidence intervals

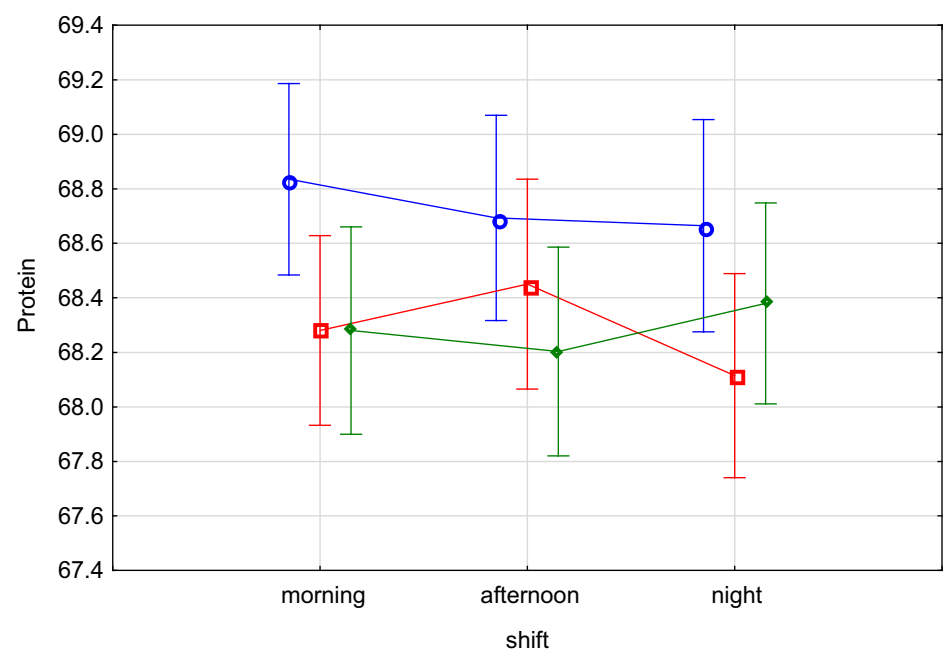

- Cooke 1 Cooker 2 $\exists$ Cooker

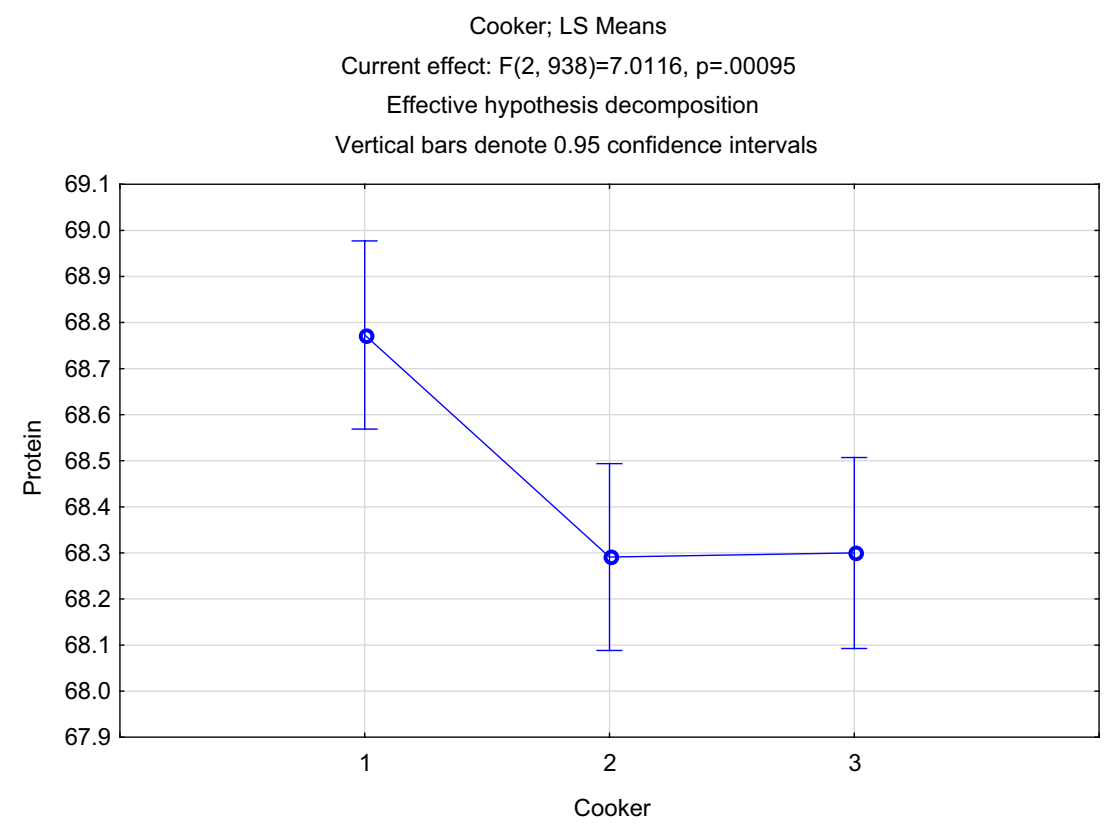

1. Process settings: the variables of PRESS PRESSURE, AUGER SPEED, and DROP TEMPERATURE have an effect on the output variables.

(a) Specifically, the main output variables of FAT and PROTEIN are dependent on PRESS PRESSURE. Achieving less variability in PRESS PRESSURE is likely to reduce the variability in FAT and PROTEIN. There may be other mechanics and hidden variables, whereby PRESS PRESSURE affects or causes the outputs, and further work could be valuable to better understand what is going on here.

(b) Tacit knowledge of operators affects rendering process. We recommend attention to practices regarding setting AUGER SPEED. The auger is the device that feeds cooked product into the 
Fig. 16 PRESS PRESSURE used in operation of plant. The differences between the SHIFTS are not statistically significant, nor between COOKERS

Fig. 17 AUGER SPEED used in operation of plant. The differences between the SHIFTS are not statistically significant, nor between COOKERS
Cooker*shift; LS Means

Current effect: $F(4,848)=.26778, p=.89874$

Effective hypothesis decomposition

Vertical bars denote 0.95 confidence intervals

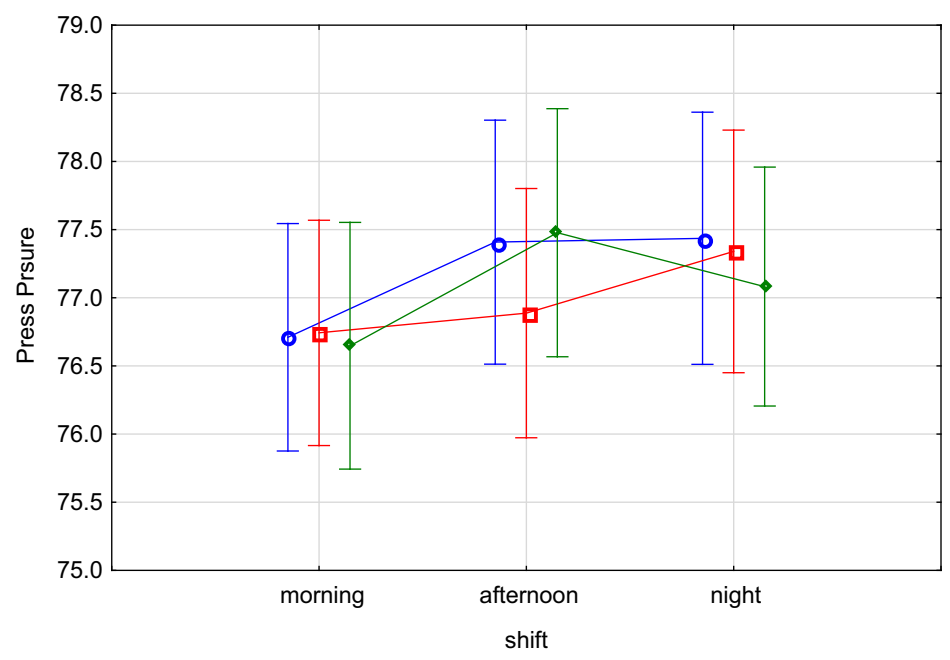

Cooker 1 프 Cooker 2 $\rightrightarrows$ Cooker

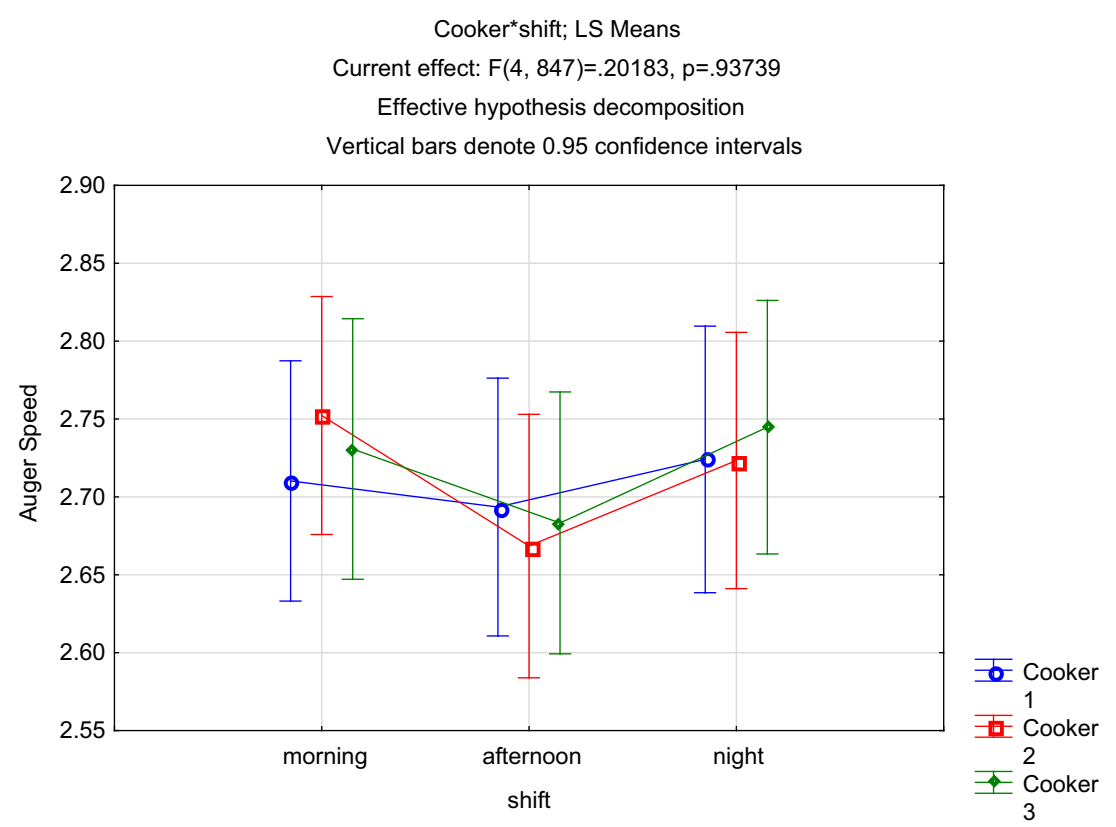

press. It is possible that the speed of this device is affecting the density of the product as it enters the press system (which has its own fixed-speed auger). In turn, this suggests that operator judgements at the unloading of the cooker may be affecting the process.

(c) An auger speed of no higher than 3 is advisable.

(d) We recommend that teamwork be encouraged so that a common organisational mental model of the plant can be developed.

(e) The rendering process has a long cycle time. Together with the relatively long time required for the laboratory tests and the multiple shifts, means that operators get little feedback on how 
Fig. 18 DROP

TEMPERATURE used in operation of plant. The differences between the SHIFTS are not statistically significant, nor between COOKERS, except that the combination of COOKER 3 on the afternoon SHIFT stands out as being at a small but significantly ( $p=0.073$ ) higher temperature. The difference is less than a degree, and it is not immediately apparent how this might affect the production process, if at all
Fig. 19 There is a reason to believe that the ASH content is entirely determined in the input material and cooking process.

The AUGER SPEED and PRESS PRESSURE are process variables from after the cooking process, and thus unlikely to be involved. They can therefore be eliminated as variables for ASH. The main factor influencing ASH is therefore the SHIFT
Cooker*shift; LS Means

Current effect: $F(4,848)=2.1471, p=.07323$

Effective hypothesis decomposition

Vertical bars denote 0.95 confidence intervals

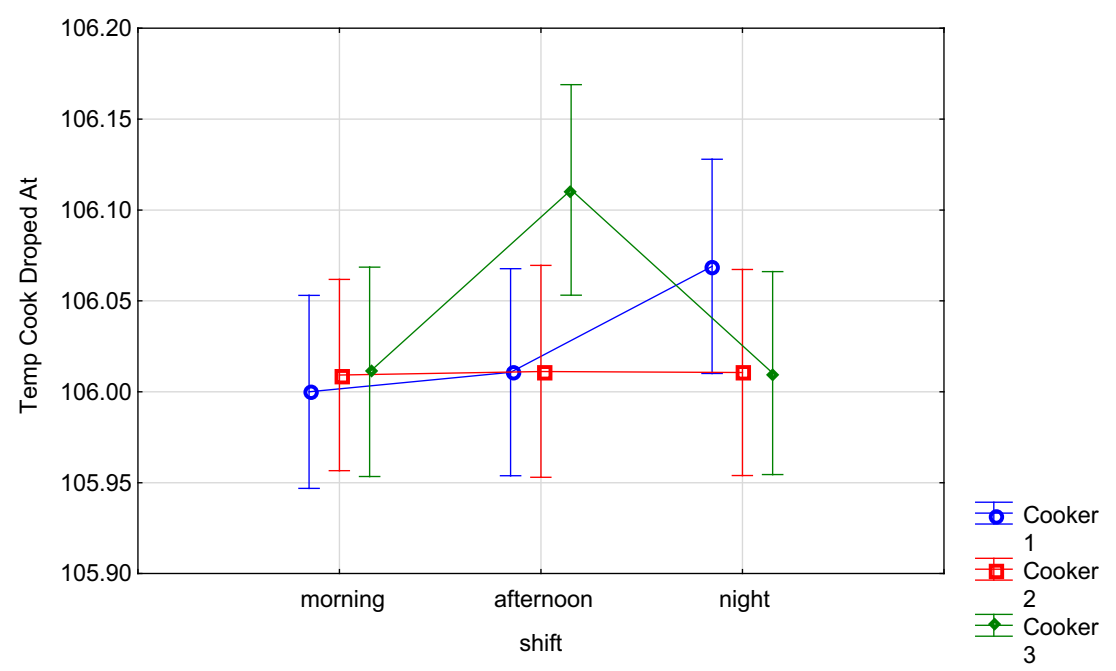

Importance plot

Dependent variable:

Ash

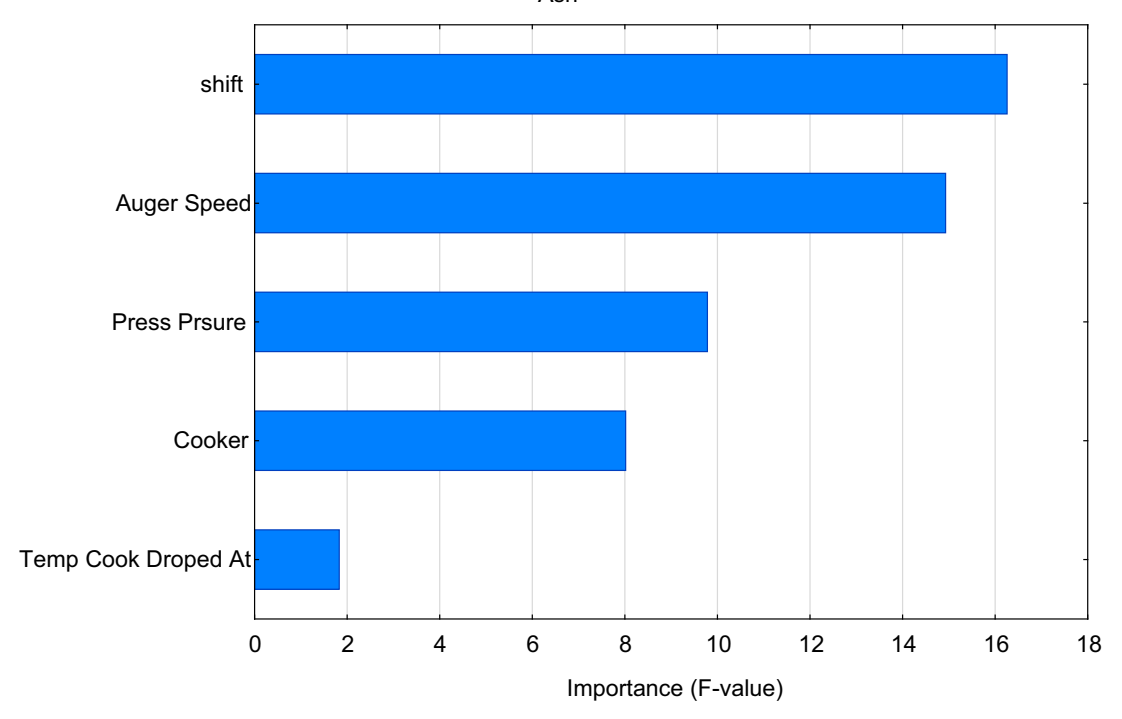

their previous efforts turned out. So, deviations are not being recognised when they occur. There is no opportunity to rework the past batch either. So, the current situation removes the motivation to reflect on the past and improve the future. We recommend that run charts or other means be set up to give operators' feedback on the success of their previous efforts (to encourage individual learning). Try to reduce assay time, to get results back to operators quicker, while they still have a chance of remembering the batch concerned. Require operators to note explanations for batches out of limits. 
Fig. 20 Main variables determining residual FAT are PRESS PRESSURE and AUGER SPEED, along with COOKER. The first two are variables measured at or immediately upstream of the FAT extraction process, and therefore of high relevance to this study. We put COOKER aside as it refers to installed plant and may be difficult to get gains in this area
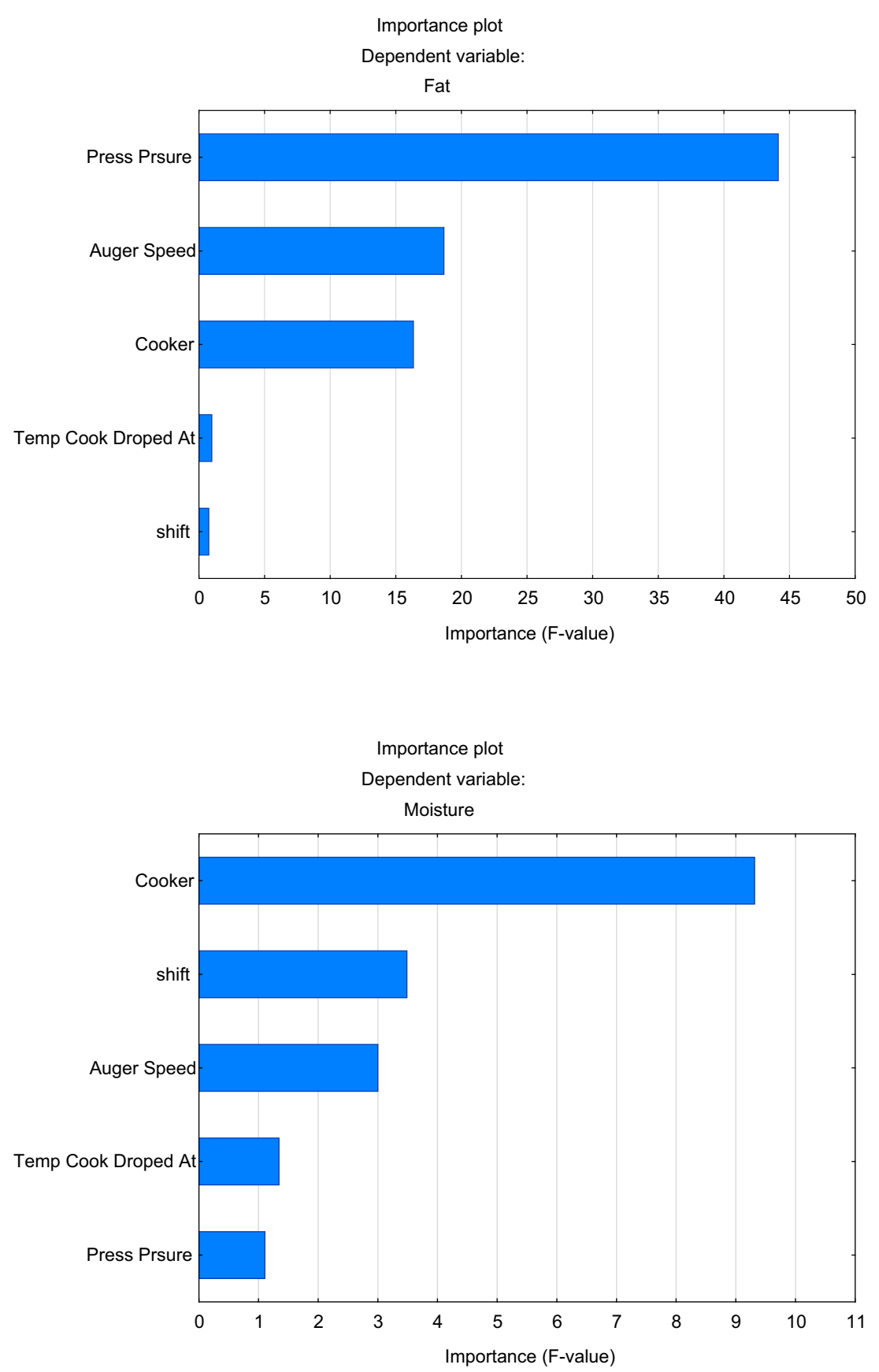

Dependent variable:

Fat
Importance plot
Fig. 21 Amount of residual MOISTURE in the meal is primarily determined by COOKER, and then secondly, by SHIFT and AUGER SPEED. We elect not to focus on COOKER at this stage, because operators do not treat the cookers differently, so this suggests it is a hardware variable and is expected to be difficult to change without changing the hardware itself (hence costly). Therefore, SHIFT and AUGER SPEED emerge as the priorities at this

stage
2. Plant variables: the COOKER (one of three) seems to determine the FAT and MOISTURE levels. Quite why is not apparent in the statistical analysis, but it does suggest that there might be something different about the COOKERS that could be worth looking into.

3. Tacit knowledge and organisational variables: a number of outcomes, especially $\mathrm{ASH}$, are dependent on 
Fig. 22 Variability in PROTEIN content is mostly made up of variability in PRESS PRESSURE, and secondly, COOKER and AUGER SPEED

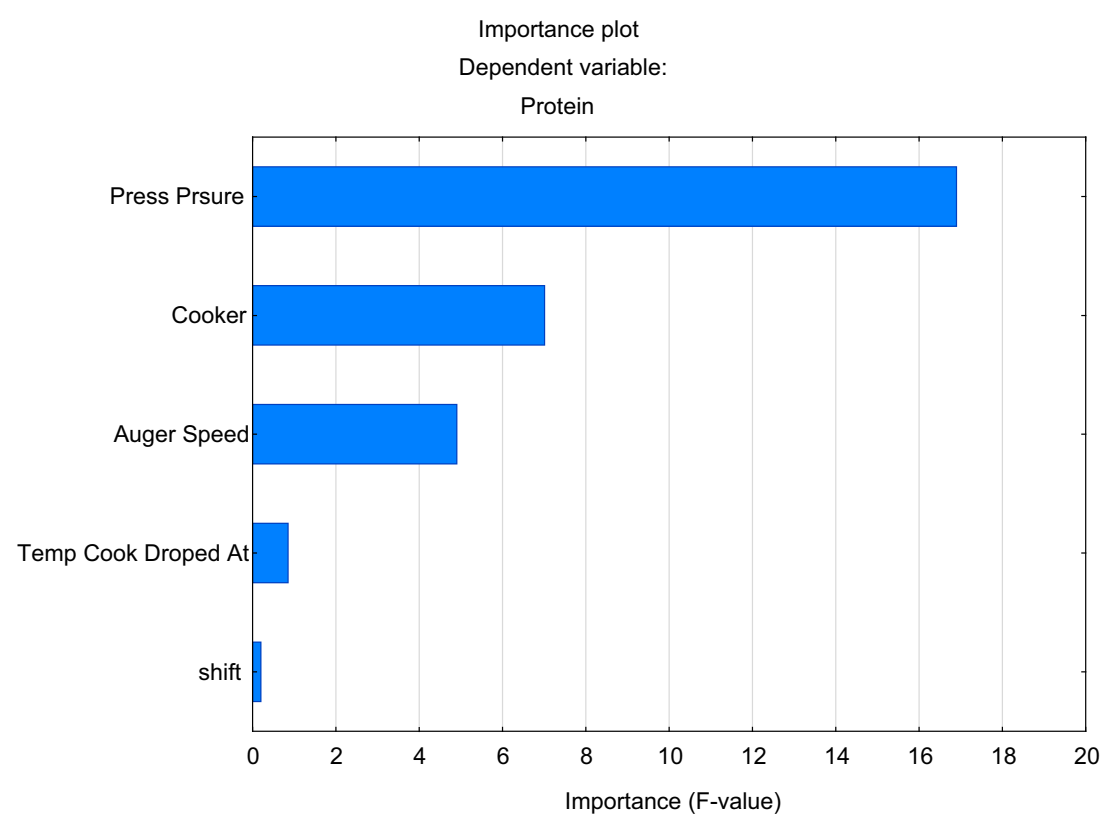

SHIFT. The same senior operators always attend the same SHIFTS. This suggests that there may be subtly different operator judgements being made depending on the SHIFT, which could be explored further.

\section{References}

Alloway Jr JA (1994) Card drop shop. Quality Progress 27(7):99-104 Coleman DW (1999) Adapting Deming's funnel experiment to a content-specific area. Simulation Gaming 30(1):8-19. doi:10. 1177/104687819903000103

D'Eredita MA, Barreto C (2006) How does tacit knowledge proliferate? An episode-based perspective. Organ Studies 27(12):1821-1841. doi:10.1177/0170840606067666

Davis W (2000) Using corrective action to make matters worse. Quality Progress 33(10):56-61

Deming WE (1986) Out of the Crisis. MIT Center for Advanced Study, Cambridge

Desouza KC (2003) Facilitating tacit knowledge exchange. Commun ACM 46(6):85-88. doi:10.1145/777313.777317

FIPS (1993) Integration definition for function modeling (IDEF0). http://www.itl.nist.gov/fipspubs/idef02.doc. Retrieved 12 Aug 2003

Georgantzas NC, Katsamakas E (2008) Tampering dynamics: SDSPC insight. Human Syst Manag 27(2):89-108. doi:10.3233/ hsm-2008-0672

Goffin K, Koners U (2011) Tacit knowledge, lessons learnt, and new product development. J Prod Innov Manage 28(2):300-318. doi:10.1111/j.1540-5885.2010.00798.x

Hamieza M, Amirreza F (2012) Challenges in Managing Tacit Knowledge: a study on difficulties in diffusion of tacit knowledge in organizations. Int J Bus Soc Sci 3(19)

Hanna MD (2010) Using a spreadsheet version of deming's funnel experiment in quality management and OM classes. Decis Sci J Innov Educ 8(1):137-142. doi:10.1111/j.1540-4609.2009.00248. $\mathrm{x}$
Jasimuddin SM (2012) Knowledge management: an interdisciplinary perspective, vol 11. World Scientific

Johannessen J-A, Olaisen J, Olsen B (2001) Mismanagement of tacit knowledge: the importance of tacit knowledge, the danger of information technology, and what to do about it. Int J Inf Manage 21(1):3-20

KBSI (2000) IDEF0 Overview. http://www.idef.com/idef0.html. Retrieved 12 Aug 2003

Krehbiel TC (1994) Tampering with a stable process. Teach Stat 16(3):75-79

MacGregor JF (1990) A different view of the funnel experiment. J Quality Technol 22(4):255-259

McNaught K, Chan A (2011) Bayesian networks in manufacturing. J Manuf Technol Manag 22(6):734-747

Nonaka I (1991) The Knowledge-Creating Company. Harvard Bus Rev 69(6):96-104

Nonaka I (1994) A dynamic theory of organizational knowledge creation. Organ Sci 5(1):14-37. doi:10.2307/2635068

Olsen T (2007) Deming's quality experiments revisited. INFORMS Trans Educ 8(1):6

Pearce A, Pons D (2013) Implementing lean practices: managing the transformation risks. J Ind Eng 790291:1-19. doi:10.1155/2013/ 790291

Perraton J, Tarrant I (2007) What does tacit knowledge actually explain? J Econ Methodol 14(3):353-370. doi:10.1080/ 13501780701562559

Polanyi M (1958) Personal knowledge: towards a post-critical phylosophy. University of Chicago Press, Chicago

Rowley J (1999) What is knowledge management? Library Manag 20(8):416-420

Schall SO (2012) Variability reduction: a statistical engineering approach to engage operations teams in process improvement. Quality Eng 24(2):264-279. doi:10.1080/08982112.2012. 641143

Schmidt K (2012) The Trouble with 'Tacit Knowledge'. Comput Support Coop Work 21(2-3):163-225. doi:10.1007/s10606-0129160-8

Shewhart WA (1931) Economic control of quality of manufactured product. D. Van Nostrand Company Inc, Lancaster 
Singer G, Ben Gal I (2007) The funnel experiment: the Markov-based SPC approach. Quality Reliab Eng Int 23(8):899-913. doi:10. 1002/qre.852

Sparks RS, Field JBF (2000) Using Deming's Funnel Experiment to Demonstrate Effects of Violating Assumptions Underlying Shewhart's Control Charts. Am Stat 54(4):291-302. doi:10. 2307/2685781

Stenmark D (2000) Leveraging tacit organizational knowledge. J Manag Inf Syst 17(3):9-24
Xie M, Del Castillo E, Goh TN, Cai DQ (2001) On the monitoring of trended and regularly adjusted processes. Int $\mathrm{J}$ Prod Res 39(16):3641-3650. doi:10.1080/00207540110064910

Xie M, Tsui KL, Goh TN, Cai DQ (2002) Process capability indices for a regularly adjusted process. Int $\mathrm{J}$ Prod Res 40(10):2367-2377. doi:10.1080/00207540210133543 